TRANSACTIONS OF THE

AMERICAN MATHEMATICAL SOCIETY

Volume 352, Number 7, Pages 3189-3209

S 0002-9947(00)02614-3

Article electronically published on March 27, 2000

\title{
$K$-THEORY OF PROJECTIVE STIEFEL MANIFOLDS
}

\author{
NELZA E. BARUFATTI AND DEREK HACON
}

\begin{abstract}
Using the Hodgkin spectral sequence we calculate $K^{*}\left(X_{m, k}\right)$, the complex $K$-theory of the projective Stiefel manifold $X_{m, k}$, for $m k$ even. For $m k$ odd, we are only able to calculate $K^{0}\left(X_{m, k}\right)$, but this is sufficient to determine the order of the complexified Hopf bundle over $X_{m, k}$.
\end{abstract}

\section{INTRODUCTION}

In this paper we extend the calculation of $K^{*}\left(X_{m, k}\right)$, the complex $K$-theory ring of projective Stiefel manifolds, begun by Antoniano, Gitler, Ucci and Zvengrowski in [2. Prior to the appearance of [2], $K^{*}\left(X_{m, k}\right)$ had been calculated for the real projective spaces $X_{m, 1}([1])$ and projective orthogonal groups ([9]).

The work of Hodgkin [10, Roux [12] and, subsequently, Antoniano, Gitler, Ucci and Zvengrowski 2] emphasized the importance of the Hodgkin spectral sequence in calculating $K^{*}(G / H)$ where $G$ is a compact Lie group with $\pi_{1}(G)$ torsion free. Hodgkin laid the foundations for later work on the $K$-theory of homogeneous spaces and showed how to calculate $K^{*}(G / H)$ in various cases of interest. In [12] Roux dealt with the case of Stiefel manifolds viewed as $\operatorname{Spin}(m) / \operatorname{Spin}(m-k)$ (see also 7]). For the projective Stiefel manifolds $X_{4 n, 2 s-1}$, the case treated in [2], the subgroup $H$ of $\operatorname{Spin}(4 n)$ is isomorphic to the group $Z / 2 \times \operatorname{Spin}(4 n-2 s+1)$.

In the general case considered in the present article $X_{m, k}=\operatorname{Spin}(m) / H$, where $H$ contains $\operatorname{Spin}(m-k)$ as a subgroup of index two but in general is not a product. To calculate $K^{*}\left(X_{m, k}\right)$ the representation ring $R H$ is computed (\$4 Theorem 2) together with the restriction homomorphism $R \operatorname{Spin}(m) \rightarrow R H$. From this it can be deduced that the Hodgkin spectral sequence collapses (\$7 Theorems 4 and 5 ) when $m k$ is even.

An important application is that the order of the complexified Hopf bundle associated to the double covering $V_{m, k} \rightarrow X_{m, k}$ may be calculated for all $m$ and $k$ (this order has been obtained in [2] only for $m=4 n$ ). Consequently, nonimmersion results for projective Stiefel manifolds may be deduced as in [3], as well as alternative (somewhat shorter) proofs for the non-parallelizability results of [2].

\section{Projective Stiefel manifolds as homogeneous spaces}

The projective Stiefel manifold $X_{m, k}$ is the quotient of the Stiefel manifold $V_{m, k}$ (of orthonormal $k$-frames in $R^{m}$ ) by the involution which takes a $k$-frame $\left\{v_{1}, \cdots, v_{k}\right\}$ to its opposite $\left\{-v_{1}, \cdots,-v_{k}\right\}$. For $1 \leq k<m$, which we henceforth assume, the special orthogonal group $S O(m)$ acts transitively on $X_{m, k}$ with

Received by the editors May 27, 1993.

1991 Mathematics Subject Classification. Primary 55N15; Secondary 55R25, 57T15. 
isotropy subgroup $K_{m, k}$ consisting of all matrices of $S O(m)$ of the form $\pm I \oplus A$, where $I=I_{k}$ is the $(k \times k)$ identity matrix and $A \in O(m-k)$.

Let $p: \operatorname{Spin}(m) \rightarrow S O(m)$ be the 2 -fold covering map. Then

$$
X_{m, k}=\operatorname{Spin}(m) / H_{m, k},
$$

where $H_{m, k}=p^{-1}\left(K_{m, k}\right)$ and $\operatorname{Spin}(m-k)$ a subgroup of index 2 of $H_{m, k}$, is generated in the Clifford algebra by the last $(m-k)$ vectors of the canonical basis $\left\{e_{1}, \cdots, e_{m}\right\}$ of $R^{m}$. We have the commutative diagram

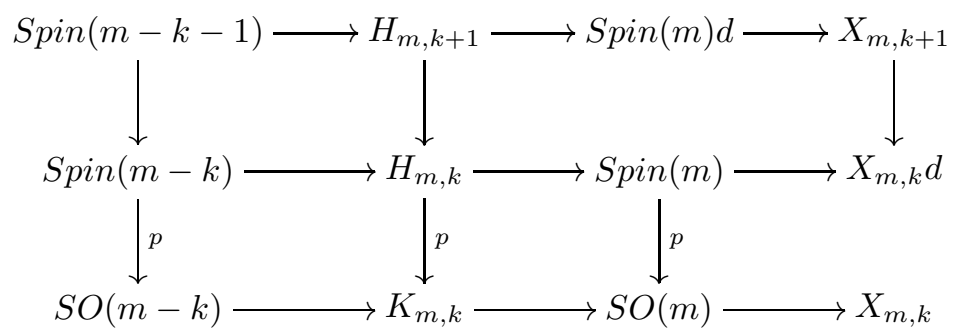

The manifolds $X_{m, k}$ and $X_{m, k+1}$ are related as follows: if $\operatorname{Spin}(m) / H_{m, k+1}$ is the corresponding expression for $X_{m, k+1}$, then $H_{m, k+1} \subset H_{m, k}$ and $\operatorname{Spin}(m-k) \cap$ $H_{m, k+1}=\operatorname{Spin}(m-k-1)$.

The following description of $H_{m, k}$ will be useful. Let $\omega$ be an element of $H_{m, k}$ that is not contained in $\operatorname{Spin}(m-k)$, so that $H_{m, k}=\operatorname{Spin}(m-k) \sqcup \omega \operatorname{Spin}(m-k)$. If at least one of $m$ or $k$ is even, $\omega$ can be chosen to lie in the center of $H_{m, k}$. In the Clifford algebra, we choose $\omega=e_{1} \cdots e_{m}$ if $m$ is even, and $\omega=e_{1} \cdots e_{k}$ if $k$ is even and $m$ odd. Note that $\omega^{2}= \pm 1$, depending on the values of $m$ and $k \bmod 4$. In general

$$
\left(e_{1} \cdots e_{r}\right)^{2}=\left\{\begin{array}{l}
+1 \text { if } r \equiv 0,3(\bmod 4), \\
-1 \text { if } r \equiv 1,2(\bmod 4) .
\end{array}\right.
$$

Let $\Omega$ be the subgroup of $H_{m, k}$ generated by $\omega$. If $\omega^{2}=+1$, then $\Omega=Z / 2$ and $H_{m, k} \approx \Omega \times \operatorname{Spin}(m-k)$. If both $m$ and $k$ are odd, $\omega$ cannot be chosen to lie in the center of $H_{m, k}$, and in this case we will choose $\omega=e_{1} \cdots e_{k+1}$. Then $\omega$ lies in the center of $H_{m, k+1}$, and $\omega^{2}= \pm 1$.

\section{The COMPlex REPRESENTAtion Ring $R \operatorname{Spin}(m)$}

Next we summarize the necessary facts on the complex representation ring of $\operatorname{Spin}(m)$ from [4 and [11].

Let $m=2 n$ or $2 n+1$, and write $T^{n}$ for the $n$-fold product $S^{1} \times \cdots \times S^{1}$, where $S^{1}$ stands as usual for the circle group in the complex plane.

Let $z_{i}$ be the character given by projection of $T^{n}$ onto the $i$ th factor. Then

$$
R T^{n}=Z\left[z_{1}, z_{1}^{-1}, \cdots, z_{n}, z_{n}^{-1}\right] .
$$

Let $\tau: T^{n} \rightarrow \operatorname{Spin}(m)$ be the homomorphism given by

$$
\tau\left(e^{i \theta_{1}}, \ldots, e^{i \theta_{n}}\right)=\left(\cos \theta_{1}+e_{1} e_{2} \sin \theta_{1}\right) \ldots\left(\cos \theta_{n}+e_{2 n-1} e_{2 n} \sin \theta_{n}\right)
$$

(product in the Clifford algebra). Then $\tau$ maps $T^{n}$ onto the maximal torus $T(n)$ of $\operatorname{Spin}(m)$. Clearly $\tau\left(z_{1}, \cdots, z_{n}\right)= \pm 1$ if and only if each $z_{i}= \pm 1$, so $\tau$ covers its image $2^{n-1}$ times. 
In $R \operatorname{Spin}(m)[t]$ we define

$$
\Lambda[t]=\sum_{i=0}^{m} t^{i} \Lambda^{i}=(1+t)^{\varepsilon} \prod_{i=1}^{n}\left(1+t z_{i}^{2}\right)\left(1+t z_{i}^{-2}\right)
$$

(where $\epsilon=0$ for $m=2 n$ and $\epsilon=1$ for $m=2 n+1$ ), and

$$
\Delta_{n}[t]=\prod_{i=1}^{n}\left(z_{i}+t z_{i}^{-1}\right) .
$$

$\Lambda[t]$ is the character of the total exterior power representation which factors through the double cover $\operatorname{Spin}(m) \rightarrow S O(m)$. As for $\Delta_{n}[t]$, putting $t=1$, we have the spinrepresentation $\Delta_{n}$ in $R \operatorname{Spin}(2 n+1)$ and the sum of the half-spin representations $\Delta_{n}^{+}+\Delta_{n}^{-}=\Delta_{n}$ in $R \operatorname{Spin}(2 n)$ with

$$
2^{\epsilon} \Delta_{n}^{2}=2^{\epsilon} \prod_{i=1}^{n}\left(z_{i}+z_{i}^{-1}\right)^{2}=2^{\epsilon} \prod_{i=1}^{n}\left(1+z_{i}^{2}\right)\left(1+z_{i}^{-2}\right)=\Lambda[1] .
$$

Putting $t=-1$, we have $\chi=\Delta_{n}^{+}-\Delta_{n}^{-}$in $\operatorname{RSpin}(2 n)$.

Let $\epsilon: R \operatorname{Spin}(m) \rightarrow Z$ be the augmentation which assigns to each representation its dimension, so that $\epsilon\left(\Lambda^{i}\right)=\left(\begin{array}{c}m \\ i\end{array}\right), \epsilon\left(\Delta_{n}\right)=2^{n}, \epsilon\left(\Delta_{n}^{ \pm}\right)=2^{n-1}$. Later on, when working with the Koszul resolution, it will be preferable to replace the exterior powers by the augmentation zero $K$-Pontrjagin classes

$$
\pi[t]=\sum_{i \geq 0} t^{i} \pi_{i}=\prod_{i=1}^{n}\left(1+t\left(z_{i}-z_{i}^{-1}\right)^{2}\right) .
$$

Note that $t^{n}$ is the highest power of $t$ occurring in this product; so $\pi_{i}=0$ for $i>n$.

One has

$$
\Lambda[t]=(1+t)^{m} \prod_{i=1}^{n}\left(1+\left(z_{i}-z_{i}^{-1}\right)^{2} \frac{t}{(1+t)^{2}}\right)=(1+t)^{m} \pi\left[\frac{t}{(1+t)^{2}}\right] .
$$

Let $\delta_{n}=\Delta_{n}-2^{n}$ and $\delta_{n}^{ \pm}=\Delta_{n}^{ \pm}-2^{n-1}$. We have (see [4] and [11])

Theorem 1. With the above notation,

(i) $\operatorname{RSpin}(2 n+1)$ is the polynomial ring $Z\left[\Lambda^{1}, \cdots, \Lambda^{n-1}, \Delta_{n}\right]$ and is also the polynomial ring $Z\left[\pi_{1}, \cdots, \pi_{n-1}, \delta_{n}\right]$. One has

$$
\Delta_{n}^{2}=\Lambda^{n}+\Lambda^{n-1}+\cdots+\Lambda^{1}+1=\pi_{n}+4 \pi_{n-1}+16 \pi_{n-2}+\cdots+2^{2 n} .
$$

(ii) $R \operatorname{Spin}(2 n)$ is the polynomial ring $Z\left[\Lambda^{1}, \cdots, \Lambda^{n-2}, \Delta_{n}^{+}, \Delta_{n}^{-}\right]$and is also the polynomial ring $Z\left[\pi_{1}, \cdots, \pi_{n-2}, \delta_{n}^{+}, \delta_{n}^{-}\right]=Z\left[\pi_{1}, \cdots, \pi_{n-2}, \chi, \delta_{n}^{+}\right]$. One has

$$
\Delta_{n}^{2}=\pi_{n}+4 \pi_{n-1}+16 \pi_{n-2}+\cdots+2^{2 n}, \Delta_{n}^{+} \Delta_{n}^{-}=\Lambda^{n-1}+\Lambda^{n-3}+\Lambda^{n-5}+\ldots
$$

and $\chi^{2}=\pi_{n}$.

(iii) Restriction Res : $R \operatorname{Spin}(2 n+1) \longrightarrow R \operatorname{Spin}(2 n)$ takes $\Lambda[t]$ to $(1+t) \Lambda[t]$, $\Delta_{n}$ to $\Delta_{n}^{+}+\Delta_{n}^{-}$, and $\pi[t]$ to $\pi[t]$.

(iv) Restriction Res : RSpin $(2 n) \longrightarrow R \operatorname{Spin}(2 n-1)$ takes $\Lambda[t]$ to $(1+t) \Lambda[t]$, $\Delta_{n}^{ \pm}$to $\Delta_{n-1}$, and $\pi[t]$ to $\pi[t]$. 


\section{THE RING $R H_{m, k}$}

Henceforth we shall write $H$ instead of $H_{m, k}$ when $m$ and $k$ are clear from the context.

We next calculate $R H$ in terms of $R \Omega$ and $R \operatorname{Spin}(m-k)$. Recall that $m=2 n$ or $2 n+1$. We let $m-k=2 c$ or $2 c+1$ and $s=n-c$. Thus, for $m=2 n, k=2 s$ or $2 s-1$, whereas, for $m=2 n+1$ we have $k=2 s$ or $2 s+1$. Note that $c=[(m-k) / 2]$ always.

4.1. $R H$ for $m-k$ odd. If $m=2 n$ is even, set $\omega=e_{1} e_{2} \cdots e_{m}$ as in $\S 2$ and define $\tilde{\omega} \in T^{n}$ to be $(i, \cdot(n) ., i)$. If $m=2 n+1$ is odd (so $k=2 s$ is even), set $\omega=e_{1} \cdots e_{k}$ as in $\S 2$ and define $\tilde{\omega} \in T^{n}$ to be $(i, .(s) ., i, 1, \ldots, 1)$. In each case $\tau(\tilde{\omega})=\omega$. Let $\tilde{\Omega}$ be the subgroup generated by $\tilde{\omega}$. Then $\tilde{\Omega} \approx Z / 4$. Since $\omega$ lies in the center of $H$, multiplication in $H$ induces an epimorphism $\tilde{\Omega} \times \operatorname{Spin}(2 c+1) \rightarrow H$ which extends the inclusion $\operatorname{Spin}(2 c+1) \subset H$ and takes $\tilde{\omega}$ to $\omega$, with kernel

$$
K=\left\{\left(\tilde{\omega}^{i}, x\right): \omega^{i}=1\right\}= \begin{cases}\left\{(1,1),\left(\tilde{\omega}^{2}, 1\right)\right\} & \text { if } \omega^{2}=1, \\ \left\{(1,1),\left(\tilde{\omega}^{2},-1\right)\right\} & \text { if } \omega^{2}=-1 .\end{cases}
$$

This induces an inclusion $R H \subset R(\tilde{\Omega} \times \operatorname{Spin}(2 c+1))=R \tilde{\Omega} \otimes R \operatorname{Spin}(2 c+1)$, and we can identify a representation in $R \tilde{\Omega} \otimes R \operatorname{Spin}(2 c+1)$ as coming from $R H$ if it is trivial on $K$.

Now, $R \tilde{\Omega}=R(Z / 4)=Z[\phi] /\left(\phi^{4}=1\right)$, where $\phi(\tilde{\omega})=i$, and $R(H / \operatorname{Spin}(2 c+1))=$ $R(Z / 2)=Z[\theta] /\left(\theta^{2}-1\right)$. The composite $\tilde{\Omega} \stackrel{\tau}{\longrightarrow} H \longrightarrow H / \operatorname{Spin}(2 c+1)$ identifies $\theta$ with $\phi^{2}$. When working in $R(\tilde{\Omega} \times \operatorname{Spin}(2 c+1))$ we will often drop the symbol $\otimes$ and write for example, $\phi, \rho, \phi \rho$ instead of $\phi \otimes 1,1 \otimes \rho, \phi \otimes \rho$. Note that $R H$ contains $\theta$ coming from $H \rightarrow H / \operatorname{Spin}(2 c+1)$ as well as the exterior powers $\Lambda^{j}=1 \otimes \Lambda^{j}$, since $\Lambda^{j}(-1)=I$. As for $\Delta_{c}$, we have $\Delta_{c}(-1)=-I$. Thus for $\omega^{2}=1$ the element $1 \otimes \Delta_{c}$ lies in $R H$ and for $\omega^{2}=-1$ the element $\phi \otimes \Delta_{c}$ lies in $R H$. In either case, we refer to this element as $\bar{\Delta}_{c}$ (note that $\bar{\Delta}_{c}=\phi^{n} \otimes \Delta_{c}$ when $m=2 n$, whereas $\bar{\Delta}_{c}=\phi^{s} \otimes \Delta_{c}$ when $m=2 n+1$ and $k=2 s$ ).

Proposition 1. Let be $m-k$ odd $(m-k=2 c+1, c \geq 0)$. Then $R H$ is the subring of $R \tilde{\Omega} \otimes R \operatorname{Spin}(2 c+1)$ generated by the elements $\theta, \Lambda^{1}, \ldots, \Lambda^{c-1}$ and $\bar{\Delta}_{c}$.

Proof. We have seen above that $R H$ contains the elements listed above. Conversely, arguing as on p.235 of [4, we prove that $R H$ is the subring fixed by a certain automorphism of $R \tilde{\Omega} \otimes R \operatorname{Spin}(2 c+1)$. The element $\left(\tilde{\omega}^{2}, \omega^{2}\right)$ lies in the center of $\tilde{\Omega} \otimes \operatorname{Spin}(2 c+1)$, and so translation by $\left(\tilde{\omega}^{2}, \omega^{2}\right)$ gives rise to a ring involution $\left(\tilde{\omega}^{2}, \omega^{2}\right)^{*}$ on $R(\tilde{\Omega} \otimes \operatorname{Spin}(2 c+1))$ which takes a typical irreducible representation $\rho$ to $\pm \rho$, the sign depending on whether $\rho\left(\tilde{\omega}^{2}, \omega^{2}\right)$ is $\pm I$. Since $\left(\tilde{\omega}^{2}, \omega^{2}\right)$ maps to 1 in $H$, this involution fixes $R H$ elementwise and corresponds to $\left(\tilde{\omega}^{2}\right)^{*} \otimes\left(\omega^{2}\right)^{*}$ on $R \tilde{\Omega} \otimes R \operatorname{Spin}(2 c+1)$. The latter fixes $\theta, \Lambda^{1}, \cdots, \Lambda^{c-1}, \bar{\Delta}_{c}$ and takes $\phi$ to $-\phi$. Hence Fix $\left(\left(\tilde{\omega}^{2}\right)^{*} \otimes\left(\omega^{2}\right)^{*}\right)$ is generated by $\theta, \Lambda^{1}, \cdots, \Lambda^{c-1}$ and $\bar{\Delta}_{c}$. It follows that $R H=\operatorname{Fix}\left(\left(\tilde{\omega}^{2}\right)^{*} \otimes\left(\omega^{2}\right)^{*}\right)$ and therefore that $R H$ is generated by the elements listed above.

4.2. $R H$ for $m$ and $k$ both even. There is a similar result when both $m$ and $k$ are even. In this case $\omega=e_{1} \cdots e_{m}$ and we define $\bar{\Delta}_{c}^{+}$and $\bar{\Delta}_{c}^{-}$in $R H$ to be $1 \otimes \Delta_{c}^{+}$and $1 \otimes \Delta_{c}^{-}$respectively if $\omega^{2}=+1$, and $\phi \otimes \Delta_{c}^{+}$and $\phi \otimes \Delta_{c}^{-}$respectively if $\omega^{2}=-1$. 
Proposition 2. Let $m$ and $k$ both be even $(m-k=2 c>0)$. Then $R H$ is the subring of $R \tilde{\Omega} \otimes R \operatorname{Spin}(2 c)$ generated by $\theta, \Lambda^{1}, \cdots, \Lambda^{c-2}, \bar{\Delta}_{c}^{+}$and $\bar{\Delta}_{c}^{-}$.

Proof. As before, the elements listed above belong to $R H$. Conversely, arguing as in Proposition 1, one has that Fix $\left(\left(\tilde{\omega}^{2}\right)^{*} \otimes\left(\omega^{2}\right)^{*}\right)$ is generated by $\theta, \Lambda^{1}, \cdots, \Lambda^{c-2}$, $\bar{\Delta}_{c}^{+}$and $\bar{\Delta}_{c}^{-}$, as we wanted.

4.3. $R H$ for $m$ and $k$ both odd. Let $m$ and $k$ be odd, so that $m-k=2 c, c>0$. In this case $X_{m, k}$ is not orientable and $H_{m, k}$ cannot be expressed as a quotient of $\tilde{\Omega} \times \operatorname{Spin}(2 c)$. However $R H_{m, k}$ can be calculated in terms of $R \operatorname{Spin}(2 c)$ and $R H_{m, k+1}$. Since $m-(k+1)=2 c-1$ is odd, $R H_{m, k+1}$ is known from Proposition [1] The argument we use is based on the calculation of $R O(2 n)$ at the end of [4]. Recall that $\omega$ is now $e_{1} \cdots e_{k+1}$, an element in the center of $H_{m, k+1}$ (but not of $\left.H_{m, k}\right)$. There is a commutative diagram of restriction homomorphims

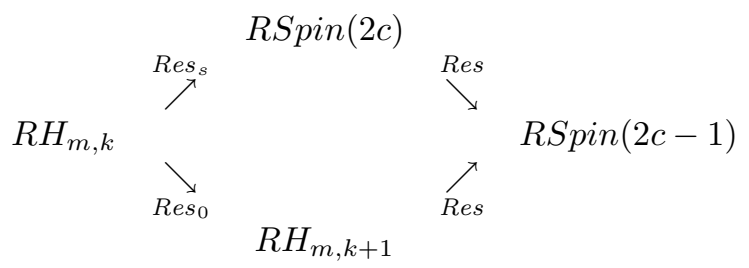

Remark 1. The reason for choosing the subgroups $\operatorname{Spin}(2 c)$ and $H_{m, k+1}$ is that, if $T(c)$ and $T(c-1)$ are "the" maximal tori of $\operatorname{Spin}(2 c)$ and $\operatorname{Spin}(2 c-1)$ respectively, then $T(c)$ and $\Omega T(c-1)=\Omega \times T(c-1)$ are Cartan subgroups of $H_{m, k}$ contained in $\operatorname{Spin}(2 c)$ and $H_{m, k+1}$ respectively.

Now $H_{m, k}$ has two connected components and so possesses exactly two conjugacy classes of Cartan subgroups. From this it follows (see [4]) that the homomorphism

$$
\left(\operatorname{Res}_{s}, \operatorname{Res}_{0}\right): R H_{m, k} \longrightarrow R \operatorname{Spin}(2 c) \oplus R H_{m, k+1} \subset R T(c) \oplus R(\Omega T(c-1))
$$

is a monomorphism. We will use the above to calculate $R H_{m, k}$ in terms of generators and relations. The generators in question are $\theta, \Lambda^{1}, \cdots, \Lambda^{c-1}$ and $\bar{\Delta}_{c}$, defined as follows. The element $\theta$ comes from $H_{m, k} \rightarrow H_{m, k} / \operatorname{Spin}(2 c)$, and $\Lambda^{1}, \cdots, \Lambda^{c-1}$ are the exterior power coming from $H_{m, k} \rightarrow H_{m, k} / \pm 1$, where $H_{m, k} / \pm 1=K_{m, k} \supset$ $S O(2 c)$. Finally, $\bar{\Delta}_{c}$ is the representation of $H_{m, k}$ induced from $\Delta_{c}^{+}$(or equivalently $\left.\Delta_{c}^{-}\right)$. The elements $\theta, \Lambda^{1}, \cdots, \Lambda^{c-1}, \bar{\Delta}_{c}$ of $R H_{m, k}$ restrict to $1, \Lambda^{1}, \cdots, \Lambda^{c-1}, \Delta_{c}$ in $R \operatorname{Spin}(2 c)$, and to $\theta, \Lambda^{1}+\theta \Lambda^{0}, \cdots, \Lambda^{c-1}+\theta \Lambda^{c-2}$ and $(1+\theta) \bar{\Delta}_{c-1}$ in $R H_{m, k+1}$ (Lemma 1 below).

Proposition 3. Let $m$ and $k$ both be odd $(m-k=2 c>0)$. Then $R H$ is generated by the elements $\theta, \Lambda^{1}, \cdots, \Lambda^{c-1}, \bar{\Delta}_{c}$ (defined above), subject only to the relations $\theta^{2}=1$ and $\theta \bar{\Delta}_{c}=\bar{\Delta}_{c}$. Furthermore, $\Lambda^{2 c-i}=\theta \Lambda^{i}$ and $\Lambda[1]=\bar{\Delta}_{c}^{2}$.

Proof. This is divided up into various lemmas.

Lemma 1. $\operatorname{Res}_{0}\left(\bar{\Delta}_{c}\right)=(1+\theta) \bar{\Delta}_{c-1}$ and $\operatorname{Res}_{0}\left(\Lambda^{i}\right)=\Lambda^{i}+\theta \Lambda^{i-1}$.

Proof. By definition $\operatorname{Res}_{s}\left(\bar{\Delta}_{c}\right)=\Delta_{c}^{+}+\Delta_{c}^{-}$, so the restriction of $\bar{\Delta}_{c}$ to $\operatorname{Spin}(2 c-1)$ is $2 \Delta_{c-1}$. From the definition of $\bar{\Delta}_{c}$ as an induced representation one has that, on $\Omega T(c-1)$, trace $\bar{\Delta}_{c}$ is zero. But $(1+\theta) \bar{\Delta}_{c-1} \in R H_{m, k+1}$ also restricts to $2 \Delta_{c-1}$ on $R \operatorname{Spin}(2 c-1)$ and has trace zero on $\Omega T(c-1)$. Hence, $\operatorname{Res}_{0}\left(\bar{\Delta}_{c}\right)=$ $(1+\theta) \bar{\Delta}_{c-1}$. As for $\Lambda^{i}$, we have that, on $\Omega T(c-1)$, the trace of $\Lambda^{i}$ is the $i$ th 
symmetric polynomial on $z_{1}^{2}, z_{1}^{-2}, \cdots, z_{c-1}^{2}, z_{c-1}^{-2}, 1$ plus the $(i-1)$ st symmetric polynomial on $z_{1}^{2} \theta, z_{1}^{-2} \theta, \cdots, z_{c-1}^{2} \theta, z_{c-1}^{-2} \theta, \theta$. Hence $\operatorname{Res}_{0}\left(\Lambda^{i}\right)=\Lambda^{i}+\theta \Lambda^{i-1}$.

Corollary 1. In $R H_{m, k}$ one has $\theta \bar{\Delta}_{c}=\bar{\Delta}_{c}$ and $\Lambda^{2 c-i}=\theta \Lambda^{i}$.

Proof. The first equation follows from the fact that $\operatorname{Res}_{0}\left(\theta \bar{\Delta}_{c}\right)=\theta(1+\theta) \bar{\Delta}_{c-1}=$ $\operatorname{Res}_{0}\left(\bar{\Delta}_{c}\right)$ (since $\theta^{2}=1$ implies $\left.\theta(1+\theta)=1+\theta\right)$ and $\operatorname{Res}_{s}\left(\theta \bar{\Delta}_{c}\right)=\operatorname{Res}_{s}\left(\bar{\Delta}_{c}\right)$. The second equation follows from the fact that $\operatorname{Res}_{s}\left(\theta \Lambda^{i}\right)=\Lambda^{i} \approx \Lambda^{2 c-i}=\operatorname{Res}_{s}\left(\Lambda^{2 c-i}\right)$ and $\operatorname{Res}_{0}\left(\theta \Lambda^{i}\right)=\theta\left(\Lambda^{i}+\theta \Lambda^{i-1}\right)=\theta \Lambda^{i}+\Lambda^{i-1} \approx \theta \Lambda^{(2 c-1)-i}+\Lambda^{(2 c-1)-(i-1)} \approx$ $\Lambda^{2 c-i}+\theta \Lambda^{2 c-i-1}=\operatorname{Res}_{0}\left(\Lambda^{2 c-i}\right)$, so that $\theta \Lambda^{i} \approx \Lambda^{2 c-i}$.

We next identify $\operatorname{Res}_{s}\left(R H_{m, k}\right)$ and $\operatorname{Res}_{0}\left(R H_{m, k}\right)$ as fixed rings of certain automorphisms of $R \operatorname{Spin}(2 c)$ and $R H_{m, k}$. To this end, consider the elements $\omega$ in the center of $R H_{m, k+1}$ and $\alpha=e_{k+1} \cdots e_{m}$ in the center of $\operatorname{Spin}(2 c)$. Then $\omega$ normalizes $\operatorname{Spin}(2 c)$ and $\alpha$ normalizes $H_{m, k+1}$, since $\alpha$ anticommutes with $\omega$.

Let $C_{\omega}: R \operatorname{Spin}(2 c) \rightarrow R \operatorname{Spin}(2 c)$ be the automorphism induced by conjugation by $\omega$, and similarly for $C_{\alpha}: R H_{m, k+1} \rightarrow R H_{m, k+1}$. Clearly $C_{\omega}$ fixes anything in the image of $R e s_{s}$, since $C_{\omega}: R H_{m, k} \rightarrow R H_{m, k}$ is the identity. Hence $C_{\omega}$ fixes $\Lambda^{1}, \cdots, \Lambda^{c-1}$. As for $\bar{\Delta}_{c}^{+}$and $\bar{\Delta}_{c}^{-}$, we note that $\omega$ normalizes the maximal torus $T(c)$ of $\operatorname{Spin}(2 c)$, and so gives rise to $C_{\omega}: R T(c) \rightarrow R T(c)$. It is easy to see that $C_{\omega}$ inverts just one $z_{j}$ (namely $z_{c}$ ) and so exchanges $\Delta_{c}^{+}$and $\Delta_{c}^{-}$.

Turning now to $C_{\alpha}: R H_{m, k+1} \rightarrow R H_{m, k+1}$, we see that $C_{\alpha}$ fixes the elements $\theta, \Lambda^{1}, \cdots, \Lambda^{c-1}$ and $(1+\theta) \bar{\Delta}_{c-1}$ in $\operatorname{Res}_{0}\left(R H_{m, k}\right)$. Since $R H_{m, k+1}$ is generated by $\theta, \Lambda^{1}, \cdots, \Lambda^{c-2}$ and $\bar{\Delta}_{c-1}$ we need only look at $C_{\alpha}\left(\bar{\Delta}_{c-1}\right)$.

Lemma 2. $C_{\alpha}\left(\bar{\Delta}_{c-1}\right)=\theta \bar{\Delta}_{c-1}$.

Proof. Since $\alpha$ belongs to the center of $\operatorname{Spin}(2 c)$, it follows that on $\operatorname{Spin}(2 c-1)$ we have $C_{\alpha}\left(\bar{\Delta}_{c-1}\right)=\bar{\Delta}_{c-1}=\theta \bar{\Delta}_{c-1}$. And, for $h \in \operatorname{Spin}(2 c-1)$, we have

$$
\begin{gathered}
C_{\alpha}\left(\bar{\Delta}_{c-1}\right)(\omega h)=\bar{\Delta}_{c-1}\left(\alpha^{-1} \omega h \alpha\right)=\bar{\Delta}_{c-1}(-\omega h)=\bar{\Delta}_{c-1}(-1) \bar{\Delta}_{c-1}(\omega h) \\
=-\bar{\Delta}_{c-1}(\omega h)=\theta(\omega h) \bar{\Delta}_{c-1}(\omega h)
\end{gathered}
$$

proving the lemma.

Let $A$ be the subring of $R H_{m, k}$ generated by $\theta, \Lambda^{1}, \cdots, \Lambda^{c-1}$ and $\bar{\Delta}_{c}$. Our objective is to prove (Lemma 4) that $R H_{m, k}=A$.

Lemma 3. $\operatorname{Res}_{s}\left(R H_{m, k}\right)=\operatorname{Res}_{s}(A)$ and $\operatorname{Res}_{0}\left(R H_{m, k}\right)=\operatorname{Res}_{0}(A)$.

Proof. First, $\operatorname{Res}_{s}(A) \subseteq \operatorname{Res}_{s}\left(R H_{m, k}\right) \subseteq F i x\left(C_{\omega}\right)$. Conversely, $R \operatorname{Spin}(2 c)=$ $Z\left[\Lambda^{1}, \cdots, \Lambda^{c-2}, \Delta_{c}^{+}, \Delta_{c}^{-}\right]$, and $C_{\omega}$ fixes $\Lambda^{1}, \cdots, \Lambda^{c-2}$ and swaps $\Delta_{c}^{+}$and $\Delta_{c}^{-}$. Hence Fix $\left(C_{\omega}\right)$ is generated by $\Lambda^{1}, \cdots, \Lambda^{c-2}, \Delta_{c}^{+} \Delta_{c}^{-}$and $\Delta_{c}^{+}+\Delta_{c}^{-}$, i.e., by $\Lambda^{1}, \cdots, \Lambda^{c-1}$ and $\bar{\Delta}_{c}$. But the latter all belong to $\operatorname{Res}_{s}(A)$. Hence $\operatorname{Res}_{s}\left(R H_{m, k}\right)=\operatorname{Res}_{s}(A)$, as required.

Next note that, as a $Z\left[\theta, \Lambda^{1}, \cdots, \Lambda^{c-1}\right]$-module, $R H_{m, k+1}$ is freely generated by 1 and $\bar{\Delta}_{c-1}$ since $\bar{\Delta}_{c-1}^{2}$ lies in $Z\left[\theta, \Lambda^{1}, \cdots, \Lambda^{c-1}\right]$ (cf.83, Theorem 1 (i)). Since $C_{\alpha}$ fixes $\theta, \Lambda^{1}, \cdots, \Lambda^{c-1}$ and sends $\bar{\Delta}_{c-1}$ to $\theta \bar{\Delta}_{c-1}$ (Lemma 2), it follows that Fix $\left(C_{\alpha}\right)$ is generated by $\theta, \Lambda^{1}, \cdots, \Lambda^{c-1}$ and $(1+\theta) \bar{\Delta}_{c-1}$. Since $\theta$ and $\Lambda^{i}+\theta \Lambda^{i-1}$ both lie in $\operatorname{Res}_{0}\left(R H_{m, k}\right)$, so does $\Lambda^{i}$. Hence $\operatorname{Res}_{0}(R H)=\operatorname{Res}_{0}(A)$.

Lemma 4. $A=R H_{m, k}$ 
Proof. Let $x \in R H_{m, k}$. By Lemma 3 there exists $y \in A$ such that $\operatorname{Res}_{s}(x)=$ $\operatorname{Res}_{s}(y)$. So we may assume that $\operatorname{Res}_{s}(x)=0$. Now consider $\operatorname{Res}_{0}(x)$. It belongs to $\operatorname{Res}_{0}\left(R H_{m, k}\right)$ which by Lemmas 1 and 3 is generated by $\theta, \Lambda^{1}, \cdots, \Lambda^{c-1}$ and $(1+\theta) \bar{\Delta}_{c-1}$. Since $\left((1+\theta) \bar{\Delta}_{c-1}\right)^{2} \in Z\left[\theta, \Lambda^{1}, \cdots, \Lambda^{c-1}\right]$, we may write $\operatorname{Res}_{0}(x)$ in the form $a+b \theta+(1+\theta) d \bar{\Delta}_{c-1}$, where $a, b, d \in Z\left[\Lambda^{1}, \cdots, \Lambda^{c-1}\right]$. But $\operatorname{ResRes}_{0}(x)=$ $\operatorname{ResRes}_{s}(x)=0$ (where in both cases Res stands for "restriction to $\operatorname{Spin}(2 c-1)$ "). Hence $a+b+2 d \Delta_{c-1}=0$ in $R \operatorname{Spin}(2 c-1)$. Now $R \operatorname{Spin}(2 c-1)=Z\left[\Lambda^{1}, \cdots, \Lambda^{c-2}\right.$, $\left.\Delta_{c-1}\right]$ is freely generated over $Z\left[\Lambda^{1}, \cdots, \Lambda^{c-1}\right]$ by 1 and $\Delta_{c-1}$. It follows that $a+b=0$ and $d=0$. Thus, $\operatorname{Res}_{0}(x)=(1-\theta) a$. Now, $\operatorname{Res}_{0}: R H_{m, k} \longrightarrow R H_{m, k+1}$ sends $\Lambda^{1}, \cdots, \Lambda^{c-1}$ to $\Lambda^{1}+\theta, \cdots, \Lambda^{c-1}+\theta \Lambda^{c-2}$ and hence sends $(1-\theta) \Lambda^{1}, \cdots$ to $(1-\theta)\left(\Lambda^{1}+\theta\right), \cdots$, which is to say $(1-\theta)\left(\Lambda^{1}-1\right), \cdots$, since $\theta^{2}=1$. Thus Res $_{0}$ sends $(1-\theta) \tilde{a}$ (where $\tilde{a}=\tilde{a}\left(\Lambda^{1}, \cdots, \Lambda^{c-1}\right)$ in $\left.R H_{m, k}\right)$ into $(1-\theta) \tilde{a}\left(\Lambda^{1}-1, \cdots\right.$, $\left.\Lambda^{c-1}-\Lambda^{c-2}\right)$. Now $\tilde{a}$ may be chosen so that $\operatorname{Res}_{0}((1-\theta) \tilde{a})=(1-\theta) a$. But clearly $\operatorname{Res}_{s}((1-\theta) \tilde{a})=0$. Recalling that $\operatorname{Res}_{s}(x)=0$, we conclude that $x=(1-\theta) \tilde{a}$, since both restrict to the same elements in $R \operatorname{Spin}(2 c)$ and $R H_{m, k+1}$. Thus $R H_{m, k}=A$, since $(1-\theta) \tilde{a} \in A$.

Finally we determine the relations in $R H$.

Lemma 5. All relations among $\theta, \Lambda^{1}, \cdots, \Lambda^{c-1}$ and $\bar{\Delta}_{c}$ in $R H$ are consequences of $\theta^{2}=1$ and $\theta \bar{\Delta}_{c}=\bar{\Delta}_{c}$.

Proof. We know that $\theta^{2}=1, \theta \bar{\Delta}_{c}=\bar{\Delta}_{c}$ and $\theta \Lambda^{i}=\Lambda^{2 c-i}$ do hold in $R H$ (Corollary 1). Suppose we have a relation among $\theta, \Lambda^{1}, \cdots, \Lambda^{c-1}$, and $\bar{\Delta}_{c}$. Using $\theta^{2}=1$ and $\theta \bar{\Delta}_{c}=\bar{\Delta}_{c}$ we may write this as $f\left(\bar{\Delta}_{c}\right)+a \theta=0$, where $f \in Z\left[\Lambda^{1}, \cdots, \Lambda^{c-1}\right][x]$ and $a \in Z\left[\Lambda^{1}, \cdots, \Lambda^{c-1}\right]$. Then $0=\operatorname{Res}_{s}(0)=f\left(\bar{\Delta}_{c}\right)+a$. But $\Lambda^{1}, \cdots, \Lambda^{c-1}, \Delta_{c}$ are algebraically independent in $R \operatorname{Spin}(2 c)$. Thus $f(x)=-a$ in $Z\left[\Lambda^{1}, \cdots, \Lambda^{c-1}\right][x]$. So, the relation is $(1-\theta) a=0$. But then $0=\operatorname{Res}_{0}(0)=(1-\theta) \operatorname{Res}_{0}(a)$. Now $a$ is a polynomial in $\Lambda^{1}, \cdots, \Lambda^{c-1}$ and as above

$$
(1-\theta) \operatorname{Res}_{0}(a)=(1-\theta) a\left(\Lambda^{1}-1, \cdots, \Lambda^{c-1}-\Lambda^{c-2}\right) .
$$

By Proposition 1 we have $a\left(\Lambda^{1}-1, \cdots, \Lambda^{c-1}-\Lambda^{c-2}\right)=0$. Since $\Lambda^{1}, \cdots, \Lambda^{c-1}$ are polynomial generators of $Z\left[\Lambda^{1}, \cdots, \Lambda^{c-1}\right]$, as are $\Lambda^{1}-1, \cdots, \Lambda^{c-1}-\Lambda^{c-2}$, we may conclude that $a=0$. So, all relations follow from $\theta^{2}=1$ and $\theta \bar{\Delta}_{c}=\bar{\Delta}_{c}$. This proves Lemma 5

This also concludes the proof of Proposition 3 .

We collect the above results on the structure of $R H$ in Theorem 2 below. In the case $m k$ even, it will be convenient to introduce Pontrjagin classes in $R H$ analogous to the ones in $R \operatorname{Spin}(m)$. The easiest way to do this is to note that, in Proposition 11 the generators $\Lambda^{1}, \cdots, \Lambda^{c-1}, \theta$, and $\bar{\Delta}_{c}$ may be replaced by $\pi_{1}, \cdots, \pi_{c-1}, y=$ $\theta-1$, and $\delta_{c}$ and similarly in Proposition 2, as in 93 . When $m$ and $k$ are odd, Pontrjagin classes are not available; this is one of the reasons why this case turns out to be much thornier than the rest; but since we will use the Koszul resolution later on for all cases, we use the augmentation zero classes $\lambda_{i}=\Lambda^{i}-\left(\begin{array}{c}2 c \\ i\end{array}\right), i=1, \cdots, 2 c$, when $m k$ is odd.

Theorem 2. (i) For $m-k=2 c+1, c>0$ ( $k=2 s$ or $k=2 s-1)$, $R H$ is generated by $\theta, \Lambda^{1}, \cdots, \Lambda^{c-1}$ and $\bar{\Delta}_{c}$ (or equivalently by $y=\theta-1, \pi_{1}, \cdots, \pi_{c-1}$, and $\delta_{c}$ ) subject only to the relation $\theta^{2}-1$ (or equivalently $y^{2}+2 y=0$ ). The element $\pi_{c}$ is given 
in terms of the generators by

$$
\begin{array}{lll}
\bar{\Delta}_{c}^{2} & =\theta^{s} \sum_{i=o}^{c} 2^{2(c-i)} \pi_{i} & \text { if } m \text { is odd }, \\
\bar{\Delta}_{c}^{2}=\theta^{n} \sum_{i=o}^{c} 2^{2(c-i)} \pi_{i} & \text { if } m \text { is even. }
\end{array}
$$

(ii) For $m$ and $k$ both even $(m-k=2 c>0, m=2 n$ and $k=2 s)$, RH is generated by $\Lambda^{1}, \cdots, \Lambda^{c-2}, \bar{\Delta}_{c}^{+}, \bar{\Delta}_{c}^{-}$and $\theta$ (or equivalently by $\pi_{1}, \cdots, \pi_{c-2}, \delta_{c}^{+}, \delta_{c}^{-}$ and $y$ ) subject only to the relation $\theta^{2}=1$ (equivalently $y^{2}+2 y=0$ ). The elements $\pi_{c}$ and $\pi_{c-1}$ are given in terms of the generators by

$$
\begin{gathered}
\bar{\Delta}_{c}^{2}=\theta^{n} \sum_{i=0}^{c} 2^{2(c-i)} \pi_{i}, \quad \bar{\Delta}_{c}^{+} \bar{\Delta}_{c}^{-}=\theta^{n} \sum_{i=0}^{c-1} 2^{2(c-1-i)} \pi_{i}, \\
\chi^{2}=\left(\delta_{c}^{+}-\delta_{c}^{-}\right)^{2}=\theta^{n} \pi_{c} .
\end{gathered}
$$

(iii) For $m$ and $k$ both odd $(m-k=2 c>0) R H$ is generated by $\theta, \Lambda^{1}, \cdots, \Lambda^{c-1}$ and $\bar{\Delta}_{c}$ (equivalently by $y, \lambda_{1}, \cdots, \lambda_{c-1}$, and $\delta_{c}$ ) subject only to the relations $\theta^{2}=1$ and $\theta \bar{\Delta}_{c}=\bar{\Delta}_{c}$ (equivalently by $y^{2}+2 y=0$ and $\left.\delta_{c} y=-2^{c} y\right)$. The element $\Lambda^{c}$ is given in terms of the generators by

$$
\bar{\Delta}_{c}^{2}=(1+\theta)\left(1+\Lambda^{1}+\ldots+\Lambda^{c-1}\right)+\Lambda^{c} .
$$

Proof. The theorem follows almost immediately from Propositions [1] 2] and 3 .

Remark 2. In addition to $y^{2}+2 y=0$, notice the following formulae, which follow from the definition of y and $\theta: y^{j}=(-2)^{j-1} y$ for $j>0, y \theta^{j}=y(1+y)^{j}=(-1)^{j} y$, for $j \geq 0,(1+y)^{2}=1,(1+\theta)^{s}=2^{s-1}(1+\theta), \theta(1+\theta)=1+\theta$ and $y(1+\theta)=0$. These will be used many times henceforth and without specific mention.

\section{The RESTRICTION Res : $R \operatorname{Spin}(m) \longrightarrow R H$}

5.1. Restriction for $m-k$ odd. Let $\tilde{\Omega}$ be as in 4.1 We have the following commutative diagram:

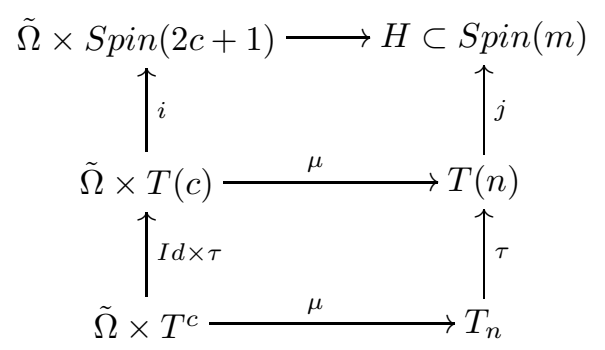

where $\mu$ stands for multiplication and $i, j$ are inclusions. Since $\omega$ lies in the center of $H$, every element of $H$ is conjugate to an element of $\tilde{\Omega} T(c)$. Hence it suffices to calculate the homomorphism $R \operatorname{Spin}(m) \rightarrow R \tilde{\Omega} \otimes R T(c)$ induced by $\mu$, or equivalently to calculate $\mu^{\#}: R T^{n} \longrightarrow R \tilde{\Omega} \otimes R T^{c}$.

Case 1. $k$ even, $m$ odd $(m=2 n+1, k=2 s, s+c=n)$. 
In this case the restriction is Res: $R \operatorname{Spin}(2 n+1) \rightarrow R H_{2 n+1,2 s}$. Hence

$$
\text { Res: } Z\left[\pi_{1}, \cdots, \pi_{n-1}, \delta_{n}\right] \rightarrow Z\left[\bar{\pi}_{1}, \cdots, \bar{\pi}_{c-1}, \delta_{c}, y\right] /\left(y^{2}+2 y\right)
$$

Here, $\bar{\pi}[t]$ denotes the $\pi[t]$ corresponding to $R H[t]$. Observing, as in 4.1, that $Z / 4 \approx \tilde{\Omega} \subset T^{n}$ is generated by $\tilde{\omega}=\left(i, ._{(s)} \cdot, i, 1 \cdots, 1\right)$ and that a generic element $\xi \in T^{c}$ has the form $\xi=\left(1, \ldots, 1, e^{i \theta_{s+1}}, \ldots, e^{i \theta_{n}}\right)$, it is easy to see that

$$
\mu^{\#}\left(z_{j}\right)= \begin{cases}\phi, & 1 \leq j \leq s, \\ z_{j}, & s+1 \leq j \leq n .\end{cases}
$$

Hence, defining $\pi^{\prime}[t]=\mu^{\#} \pi[t]$, we have

$$
\begin{aligned}
\pi^{\prime}[t] & =\mu^{\#} \prod_{j=1}^{n}\left(1+t\left(z_{j}-z_{j}^{-1}\right)^{2}\right) \\
& =\prod_{j=1}^{s}\left(1+t\left(\phi-\phi^{-1}\right)^{2}\right) \prod_{j=s+1}^{n}\left(1+t\left(z_{j}-z_{j}^{-1}\right)^{2}\right) \\
& =(1+2 t y)^{s} \bar{\pi}[t] \quad\left(\text { since }\left(\phi-\phi^{-1}\right)^{2}=2 \theta-2=2 y\right) .
\end{aligned}
$$

It follows that

$$
\bar{\pi}[t]=(1+2 t y)^{-s} \pi^{\prime}[t],
$$

so that $\bar{\pi}_{i}$ is a linear combination of $\pi_{1}^{\prime}, \cdots, \pi_{i}^{\prime}$ with coefficients in $Z[y]$, for $i=$ $1, \cdots, c$.

Next, consider $\Delta_{n} \in R \operatorname{Spin}(2 n+1)$. Since $\Delta_{n}=\prod_{j=1}^{n}\left(z_{j}+z_{j}^{-1}\right)$, we have

$$
\begin{aligned}
j^{\#}\left(\Delta_{n}\right) & =\prod_{j=1}^{s}\left(\phi+\phi^{-1}\right) \prod_{j=s+1}^{n}\left(z_{j}+z_{j}^{-1}\right) \\
& =\left\{\begin{array}{ll}
\phi(1+\theta)^{s} \Delta_{c} & \text { if } s \text { odd } \\
(1+\theta)^{s} \Delta_{c} & \text { if } s \text { even }
\end{array}\right\}=(1+\theta)^{s} \bar{\Delta}_{c} .
\end{aligned}
$$

So, we obtain

$$
\operatorname{Res}\left(\Delta_{n}\right)=2^{s-1}(2+y) \bar{\Delta}_{c} .
$$

From (1) and (3), we have

Proposition 4. For $m=2 n+1$ and $k=2 s$, Res $: R \operatorname{Spin}(2 n+1) \rightarrow R H$ is given by

$$
\begin{aligned}
& \operatorname{Res}(\pi[t])=\pi^{\prime}[t]=(1+2 t y)^{s} \bar{\pi}[t] \\
& \operatorname{Res}\left(\delta_{n}\right)=2^{s-1}(2+y) \delta_{c}+2^{n-1} y .
\end{aligned}
$$

Case 2. $k$ odd, $m$ even $(m=2 n, k=2 s-1)$.

In this case the restriction is Res: $R \operatorname{Spin}(2 n) \rightarrow R H_{2 n, 2 s-1}$. Hence

$$
\text { Res: } Z\left[\pi_{1}, \cdots, \pi_{n-2}, \delta_{n}^{+}, \chi_{n}\right] \rightarrow Z\left[\bar{\pi}_{1}, \cdots, \bar{\pi}_{c-1}, \delta_{c}, y\right] /\left(y^{2}+2 y\right) .
$$

This time $\tilde{\omega}=(i, \cdot(n) ., i)$ and $\xi=\left(1, \cdots, 1, e^{i \theta_{s+1}}, \cdots, e^{i \theta_{n}}\right)$ imply

$$
\mu^{\#}\left(z_{j}\right)= \begin{cases}\phi, & 1 \leq j \leq s, \\ \phi z_{j}, & s+1 \leq j \leq n .\end{cases}
$$


Hence

$$
\begin{aligned}
\pi^{\prime}(t) & =\mu^{\#}(\pi[t])=\mu^{\#}\left(\prod_{j=1}^{n}\left[1+t\left(z_{j}-z_{j}^{-1}\right)^{2}\right]\right. \\
& =\prod_{j=1}^{s}\left[1+t\left(\phi-\phi^{-1}\right)^{2}\right] \prod_{j=s+1}^{n}\left[1+t\left(\phi z_{j}-\left(\phi z_{j}\right)^{-1}\right)^{2}\right] \\
& =(1+2 t y)^{s} \prod_{j=s+1}^{n}\left[1+2 t(\theta-1)+t \theta\left(z_{j}-z_{j}^{-1}\right)^{2}\right] \\
& =(1+2 t y)^{n} \prod_{j=s+1}^{n}\left[1+\left(z_{j}-z_{j}^{-1}\right)^{2} \frac{t \theta}{(1+2 t y)}\right] .
\end{aligned}
$$

Therefore,

$$
\pi^{\prime}[t]=(1+2 t y)^{n} \bar{\pi}[u]
$$

where $u=u(t)=t(1+y) /(1+2 t y)$. Since $(1+2 t y)(1+2 u y)=1$ one has $u(u(t))=1$, so that

$$
\bar{\pi}[t]=(1+2 t y)^{n} \pi^{\prime}[u],
$$

from which we conclude as before that $\bar{\pi}_{i}$ is a linear combination of $\pi_{1}^{\prime}, \cdots, \pi_{i}^{\prime}$ with coefficients in $Z[y], i=1, \cdots, c$.

Next, we have (in $R \operatorname{Spin}(2 n)) \Delta_{n}^{+}$and $\Delta_{n}^{-}$. If we write, as in $\$ 3 \Delta_{n}[t]=$ $\prod_{j=1}^{n}\left(z_{j}+t z_{j}^{-1}\right)$, we have $\Delta_{n}[t]=\Delta_{n}^{+}+t \Delta_{n}^{-}$for $t= \pm 1$. Hence, for $t= \pm 1$,

$$
\begin{aligned}
j^{\#}\left(\Delta_{n}[t]\right) & =\prod_{j=1}^{s}\left(\phi+t \phi^{-1}\right) \prod_{j=s+1}^{n}\left(\phi z_{j}+t \phi^{-1} z_{j}^{-1}\right) \\
& =(1+t \theta)^{s} \phi^{n} \prod_{j=s+1}^{n}\left(z_{j}+t \theta z_{j}^{-1}\right) \\
& =\phi^{n}(1+t \theta)^{s} \Delta_{c}[t \theta]=2^{s-1}(1+t \theta) \phi^{n} \Delta_{c}[t \theta],
\end{aligned}
$$

from which it follows that

$$
\operatorname{Res}\left(\Delta_{n}^{+}\right)=2^{s-1} \bar{\Delta}_{c} \text { and } \operatorname{Res}\left(\Delta_{n}^{-}\right)=2^{s-1} \theta \bar{\Delta}_{c} .
$$

From (4) and (6) we have

Proposition 5. For $m=2 n$ and $k=2 s-1$, Res: $R \operatorname{Spin}(2 n) \rightarrow R H$ is given by

$$
\begin{aligned}
& \operatorname{Res}(\pi[t])=\pi^{\prime}[t]=(1+2 t y)^{n} \bar{\pi}[t(1+y) /(1+2 t y)], \\
& \operatorname{Res}\left(\delta_{n}^{+}\right)=2^{s-1} \delta_{c}, \\
& \operatorname{Res}\left(\chi_{n}\right)=-2^{s-1} \delta_{c} y-2^{n-1} y .
\end{aligned}
$$

5.2. Restriction for $m$ and $k$ both even. Next $m=2 n, k=2 s$ and as before $c=n-s$. The restriction is given by Res: $R \operatorname{Spin}(2 n) \rightarrow R H_{2 n, 2 s}$. Hence

$$
\text { Res: } Z\left[\pi_{1}, \cdots, \pi_{n-2}, \delta_{n}^{+}, \chi_{n}\right] \rightarrow Z\left[\bar{\pi}_{1}, \cdots, \bar{\pi}_{c-2}, \delta_{c}^{+}, \delta_{c}, y\right] /\left(y^{2}+2 y\right) .
$$

As in 5.1 case 2 , we have

$$
\begin{gathered}
\pi^{\prime}[t]=(1+2 t y)^{n} \bar{\pi}[u], \\
\operatorname{Res}\left(\Delta_{n}^{+}\right)=2^{s-1} \bar{\Delta}_{c} \text { and } \operatorname{Res}\left(\Delta_{n}^{-}\right)=2^{s-1} \theta \bar{\Delta}_{c}
\end{gathered}
$$


(here $\bar{\Delta}_{c}=\bar{\Delta}_{c}^{+}+\bar{\Delta}_{c}^{-}$). Hence, from $(7)$ and (8) we have

Proposition 6. For $m=2 n$ and $k=2 s$, Res:RSpin $(2 n) \rightarrow R H$ is given by

$$
\begin{aligned}
& \operatorname{Res}(\pi[t])=\pi^{\prime}[t]=(1+2 t y)^{n} \bar{\pi}[t(1+y) /(1+2 t y)], \\
& \operatorname{Res}\left(\delta_{n}^{+}\right)=2^{s-1} \delta_{c}, \quad\left(\delta_{c}=\delta_{c}^{+}+\delta_{c}^{-}\right), \\
& \operatorname{Res}\left(\chi_{n}\right)=-2^{s-1} \delta_{c} y-2^{n-1} y .
\end{aligned}
$$

5.3. Restriction for $m$ and $k$ both odd. Here $m=2 n+1, k=2 s+1$. We write $\bar{\Lambda}[t]$ for the corresponding $\Lambda[t]$ to $R H_{m, k}[t]$. The restriction is given by Res:RSpin $(2 n+1) \rightarrow R H_{2 n+1,2 s+1}$. Hence

$$
\text { Res : } Z\left[\Lambda^{1}, \cdots, \Lambda^{n-1}, \Delta_{n}\right] \rightarrow Z\left[\bar{\Lambda}^{1}, \cdots, \bar{\Lambda}^{c-1}, \bar{\Delta}_{c}, \theta\right] /\left(\theta^{2}-1, \theta \bar{\Delta}_{c}-\bar{\Delta}_{c}\right) .
$$

We may identify $\operatorname{Res}(x), x \in R \operatorname{Spin}(2 n+1)$, by calculating the restriction of $x$ to $R \operatorname{Spin}(2 c)$ and to $R H_{m, k+1}$. In the case of $R H_{m, k+1}$, going back to 5.1, case 1, where Res:RSpin $(2 n+1) \rightarrow R H_{m, k+1}$, we have (note $k+1=2(s+1)$ ) that

$$
\begin{aligned}
\operatorname{Res}(\Lambda[t]) & =\mu^{\#}(1+t) \prod_{i=1}^{n}\left(1+t z_{i}^{2}\right)\left(1+t z_{i}^{-2}\right) \\
& =(1+t) \prod_{j=1}^{s+1}(1+t \theta)\left(1+t \theta^{-1}\right) \prod_{j=s+2}^{n}\left(1+t z_{j}^{2}\right)\left(1+t z_{j}^{-2}\right) \\
& =(1+t \theta)^{2 s+2} \hat{\Lambda}[t]
\end{aligned}
$$

$\left(\hat{\Lambda}[t]\right.$ denotes the $\Lambda[t]$ corresponding to $\left.R H_{m, k+1}\right)$ and $\operatorname{Res}\left(\Delta_{n}\right)=2^{s}(\theta+1) \bar{\Delta}_{c-1}$.

Now, $\Lambda[t]$ restricts to $(1+t)^{2 s+1} \Lambda[t]$ in $R \operatorname{Spin}(2 c)[t]$, and from 4.3 we have that $\operatorname{Res}_{0}(\Lambda[t])=(1+t \theta) \hat{\Lambda}[t]$ and $\operatorname{Res}_{s}(\bar{\Lambda}[t])=\Lambda[t] \in \operatorname{RSpin}(2 c)[t]$. Hence

$$
\operatorname{Res}(\Lambda[t])=\Lambda^{\prime}[t]=(1+t \theta)^{k} \bar{\Lambda}[t] .
$$

The element $\Delta_{n} \in R \operatorname{Spin}(2 n+1)$ restricts to $2^{s} \Delta_{c} \in R \operatorname{Spin}(2 c)$. But $\operatorname{Res}_{s}\left(\bar{\Delta}_{c}\right)$ $=\Delta_{c}$ and $\operatorname{Res}_{0}\left(\bar{\Delta}_{c}\right)=(1+\theta) \bar{\Delta}_{c-1}$. Hence,

$$
\operatorname{Res}\left(\Delta_{n}\right)=2^{s} \bar{\Delta}_{c} .
$$

From (9) and (10) we have

Proposition 7. For $m=2 n+1, k=2 s+1$, Res:RSpin $(2 n+1) \rightarrow R H$ is given by

$$
\begin{aligned}
& \operatorname{Res}(\Lambda[t])=\Lambda^{\prime}[t]=(1+t \theta)^{k} \bar{\Lambda}[t], \\
& \operatorname{Res}\left(\Delta_{n}\right)=2^{s} \bar{\Delta}_{c} .
\end{aligned}
$$

\section{The Hodgkin spectral sequence}

6.1. The theorem. Let $G$ be a compact, connected Lie group with $\pi_{1}(G)$ torsionfree and $H$ a closed subgroup of $G$. The Hodgkin spectral sequence calculates the complex $K$-theory of $G / H$ in terms of $R G$ and $R H$. The result is the following (cf. [10], [12]).

Theorem 3. Given $G$ and $H$ as above, there is a strongly convergent sequence $E_{r}(G / H)$ with the following three properties:

(i) As an algebra, $E_{2}^{p}(G / H)=\operatorname{Tor}_{R G}^{p}(R H ; Z)$,

(ii) The differential $d_{r}: E_{r}^{p-r} \rightarrow E_{r}^{p}$ is zero for $r$ even, 
(iii) $E_{\infty}^{*}(G / H)$ is the graded algebra associated to a negative filtration of $K^{*}(G / H)$ compatible with its multiplication

$$
\begin{gathered}
F^{p} K^{*} \otimes F^{q} K^{*} \rightarrow F^{p+q} K^{*}, \\
F^{p} K^{*}=F_{-p} K^{*}=\tilde{F}^{2 p} K^{0} \oplus \tilde{F}^{2 p+1} K .
\end{gathered}
$$

Notes: 1. $R G, R H$ and $R\{1\}=Z$ are trivially $Z / 2$-graded by $R^{0}=R$ and $R^{1}=0$.

2. If $\operatorname{Tor}_{R G}^{*}(R H, \mathrm{Z})$ is generated as an algebra by its elements of degree $\leq 2$, then the Hodgkin spectral sequence collapses. Hence in this case we have two isomorphisms

$$
\begin{gathered}
E_{2}^{0,0}=R H \otimes_{R G} Z \stackrel{\approx}{\longrightarrow} E_{\infty}^{0,0}=F_{0} K^{0} \\
E_{2}^{-1,0}=\operatorname{Tor}_{R G}^{-1}(R H ; Z) \stackrel{\approx}{\longrightarrow} E_{\infty}^{-1,0}=F_{-1} K^{-1} .
\end{gathered}
$$

6.2. Some consequences. Let $G$ and $H$ be as above, and let $R e s: R G \rightarrow R H$ be the homomorphism induced by inclusion.

As before, $\epsilon_{G}: R G \rightarrow R\{1\}=Z$ is the augmentation which assigns to each representation its dimension. We will suppose that $R G=Z\left[\gamma_{1}, \cdots, \gamma_{n}\right]$ with $\epsilon_{G}\left(\gamma_{i}\right)=0$, $i=1, \cdots, n$ (if $\epsilon_{G}\left(\gamma_{i}\right) \neq 0$ we take $\tilde{\gamma}_{i}=\gamma_{i}-\epsilon_{G}\left(\gamma_{i}\right)$ ), and $R H$ is generated by $h_{1}, \ldots, h_{m}$ with $\epsilon_{H}\left(h_{j}\right)=0, j=1, \cdots, m$.

Let $\Gamma=Z\left[\gamma_{1}, \cdots, \gamma_{r}\right], 1 \leq r \leq m$. We state the following straightforward applications of the change of rings theorem and Koszul resolution (cf. [5] and [12]):

1. If $R H$ is a free (or more generally flat) $\Gamma$-module and $\operatorname{Res}\left(\gamma_{i}\right)=h_{i}, i=$ $1, \cdots, r$, it follows that $\operatorname{Tor}_{R G}^{*}(R H ; Z)=\operatorname{Tor}_{A}^{*}(B ; Z)$, where

$$
A=R G /\left(\gamma_{1}, \cdots, \gamma_{r}\right), \quad B=R H /\left(h_{1}, \cdots, h_{r}\right)
$$

and the $A$-module structure of $B$ (which we call Res/A, or Res by a small abuse of notation) is induced by Res.

2. If $\tau_{1}, \cdots, \tau_{s} \in A$ and $(\operatorname{Res} / A)\left(\tau_{i}\right)=0,1 \leq i \leq s$, then, setting $A^{\prime}=$ $A /\left(\tau_{1}, \cdots, \tau_{s}\right), B$ is an $A^{\prime}$-module via $\operatorname{Res} / A$ and

$$
\operatorname{Tor}_{A}^{*}(B ; Z) \approx \Lambda_{Z}^{*}\left[t_{1}, \cdots, t_{s}\right] \otimes \operatorname{Tor}_{A^{\prime}}^{*}(B ; Z),
$$

where $\Lambda_{Z}^{*}\left[t_{1}, \cdots, t_{s}\right]$ denotes the graded exterior algebra with generators $t_{i}$ of degree 1 ,

3. If $R H$ is a trivial $R G$-module, then

$$
\operatorname{Tor}_{R G}^{*}(R H ; Z) \approx \Lambda_{R G}^{*}\left[t_{1}, \cdots . t_{n}\right]
$$

where $t_{1}, \cdots, t_{n}$ are of degree 1 .

\section{The COMPlex $K$-THEORY OF $X_{m, k}$ FOR $m k$ EVEN}

Now that $R H$ and the restriction $\operatorname{Res} R \operatorname{Spin}(m) \rightarrow R H$ are known, we are in position to determine $K^{*}\left(X_{m, k}\right)$ for $m k$ even. To do this we must determine the structure of the ring $B$ defined in 6 , as well as the restriction $A \rightarrow B$, so as to be able to apply the Koszul resolution. 
7.1. Structure of $B$. From $\$$ (5) relations (1), (2), (4), (5) and (7) (cases where $m k$ is even), we see that $\pi_{1}^{\prime}, \cdots, \pi_{c}^{\prime}$ can be taken as generators for $R H_{m, k}$ instead of $\bar{\pi}_{1}, \cdots, \bar{\pi}_{c}$. Since $\operatorname{Res}\left(\pi_{i}\right)=\pi_{i}^{\prime}, i=1, \cdots, c$ and $R H$ is a free $Z\left[\pi_{1}, \cdots, \pi_{c}\right]$-module it follows from 6.2 (1) that

$$
\operatorname{Tor}_{R \operatorname{Spin}(m)}^{*}(R H ; Z) \approx \operatorname{Tor}_{A}^{*}(B ; Z),
$$

where

$$
A=R \operatorname{Spin}(m) /\left(\pi_{1}, \cdots, \pi_{c}\right), \quad B=R H /\left(\pi_{1}^{\prime}, \cdots, \pi_{c}^{\prime}\right)
$$

(unfortunately, for $m$ and $k$ both odd $R H$ is not a free $Z\left[\lambda_{1}, \cdots, \lambda_{c}\right]$-module).

The homomorphism Res induces an algebra homomorphism Res/A :A $\rightarrow B$ that makes $B$ an $A$-algebra. While $A$ is still a polynomial algebra (on $c$ fewer generators), the structure of $B$ is more complicated as we now see.

From $95,(1),(5)$ and (7) we have

$$
\bar{\pi}[t]= \begin{cases}(1+2 t y)^{-s} \pi^{\prime}[t] & (m=2 n+1), \\ (1+2 t y)^{n} \pi^{\prime}[u] & (m=2 n),\end{cases}
$$

where $u=t(1+y) /(1+2 t y)$.

Now $\bar{\pi}_{i}=0$ for $i>c$, and in $B$, we have $\pi_{i}^{\prime}=0$ for $1 \leq i \leq c\left(\pi_{0}^{\prime}=1\right)$. So, for $1 \leq i \leq c$, in $B$ we have:

$$
\bar{\pi}[t]= \begin{cases}T_{c}(1+2 t y)^{-s} & (m=2 n+1), \\ T_{c}(1+2 t y)^{n} & (m=2 n),\end{cases}
$$

where $T_{c}$ is truncation defined by $T_{c}\left(\sum_{i \geq 0} a_{i} t^{i}\right)=\sum_{i=0}^{c} a_{i} t^{i}$. Thus,

$$
\bar{\pi}_{i}=\left\{\begin{array}{l}
(-1)^{i-1}\left(\begin{array}{c}
n \\
i
\end{array}\right) 2^{2 i-1} y, \\
-\left(\begin{array}{c}
s+i-1 \\
i
\end{array}\right) 2^{2 i-1} y,
\end{array} \quad 1 \leq i \leq c,\right.
$$

recalling that $y^{2}=-2 y$ and the well known identity $\left(\begin{array}{c}-s \\ i\end{array}\right)=(-1)^{i}\left(\begin{array}{c}s+i-1 \\ i\end{array}\right)$ (cf. [8]).

Substituting $u$ for $t$ in the second equation of (12) and using (4), we get

$$
\pi^{\prime}[t]=(1+2 t y)^{n} \sum_{i \geq c}\left(\begin{array}{c}
n \\
i
\end{array}\right)(2 u y)^{i} .
$$

We write $\bar{f}(t)$ for $f(-t /(1+t))$. Observe that $\overline{f g}=\bar{f} \bar{g}$ and $\overline{f+g}=\bar{f}+\bar{g}$. Then, $f(2 u y)=\bar{f}(2 t y)$. Hence $\pi^{\prime}[t]=F(2 t y)$, where $F(t)=(1+t)^{n} \overline{T_{c}(1+t)^{n}}$.

Lemma 6. With the above notation

$$
\begin{aligned}
& \text { (i) }(1+t)^{s} T_{c}(1+t)^{-s}=(1+t)^{n} \overline{T_{c}(1+t)^{n}}, \\
& \text { (ii) }(1+t)^{s} T_{c}(1+t)^{-s}=(-1)^{c} \sum_{i=0}^{c}\left(\begin{array}{c}
n \\
i
\end{array}\right)\left(\begin{array}{c}
i-1 \\
c
\end{array}\right) t^{i} .
\end{aligned}
$$

Proof. Fix $s \geq 0$. Both results are proved by induction on $c$ starting with $c=0$ where both sides of the equations are $(1+t)^{s}$, since $c=0$ implies $n=s$. The inductive steps in (i) and (ii) are obtained by using the elementary identities:

$$
\begin{gathered}
\left(\begin{array}{c}
i+1 \\
j
\end{array}\right)=\left(\begin{array}{c}
i \\
j
\end{array}\right)+\left(\begin{array}{c}
i \\
j-1
\end{array}\right), \quad\left(\begin{array}{l}
i \\
j
\end{array}\right)=(-1)^{j}\left(\begin{array}{c}
j-i-1 \\
j
\end{array}\right), \\
\left(\begin{array}{c}
n \\
i
\end{array}\right)\left(\begin{array}{c}
i \\
j
\end{array}\right)=\left(\begin{array}{c}
n \\
j
\end{array}\right)\left(\begin{array}{l}
n-j \\
i-j
\end{array}\right)
\end{gathered}
$$


valid for all $i, j$ and $n \geq 0$ (see [8]). Specifically, for (i) the inductive step is

$$
\begin{aligned}
& (1+t)^{n+1} \overline{T_{c+1}(1+t)^{n+1}}-(1+t)^{n} \overline{T_{c}(1+t)^{n}} \\
& \left.=(1+t)^{n+1} \overline{\left(\overline{T_{c}(1+t)^{n+1}}-(1+t) T_{c}(1+y)^{n}\right.}\right) \\
& \left.=(1+t)^{n+1} \overline{\left(\begin{array}{c}
n \\
c+1
\end{array}\right) t^{c+1}} \text { (since } t T_{c}(f)=T_{c+1}(t f)\right) \\
& =(1+t)^{n+1}\left(\begin{array}{c}
n \\
c+1
\end{array}\right)\left(\frac{-t}{1+t}\right)^{c+1} \\
& =(-1)^{c+1}\left(\begin{array}{c}
n \\
c+1
\end{array}\right)(1+t)^{s} t^{c+1}=(1+t)^{s}\left(\begin{array}{c}
-s \\
c+1
\end{array}\right) t^{c+1} \\
& =(1+t)^{s} T_{c+1}(1+t)^{-s}-(1+t)^{s} T_{c}(1+t)^{-s},
\end{aligned}
$$

whereas for (ii) the inductive step is: consider

$$
(-1)^{c+1} \sum_{i=0}^{n}\left(\begin{array}{c}
n \\
i
\end{array}\right)\left(\begin{array}{c}
i-1 \\
c
\end{array}\right) t^{i}-(-1)^{c} \sum_{i=0}^{n}\left(\begin{array}{c}
n \\
i
\end{array}\right)\left(\begin{array}{c}
i-1 \\
c
\end{array}\right) t^{i} .
$$

Using the elementary identities above we have

$$
\left(\begin{array}{c}
n \\
i
\end{array}\right)\left(\begin{array}{c}
i-1 \\
c
\end{array}\right)+\left(\begin{array}{c}
n+1 \\
i
\end{array}\right)\left(\begin{array}{c}
i-1 \\
c+1
\end{array}\right)=(-1)^{c+1}\left(\begin{array}{c}
-s \\
c+1
\end{array}\right)\left(\begin{array}{c}
s \\
i-c-1
\end{array}\right)
$$

which is equal to the coefficient of $t^{i}$ in $(-1)^{c+1}\left(\begin{array}{c}-s \\ c+1\end{array}\right)(1+t)^{s} t^{c+1}$.

Applying Lemma 6 to the expression (15) for $\pi^{\prime}[t]$ for $m$ even and (13) for $m$ odd, we conclude that in $B$, for $m$ even or odd,

$$
\pi_{i}^{\prime}=(-1)^{c+1+i} 2^{2 i-1}\left(\begin{array}{c}
n \\
i
\end{array}\right)\left(\begin{array}{c}
i-1 \\
c
\end{array}\right) y, \quad i>c .
$$

In addition to this explicit formula for $\pi_{i}^{\prime}, i>c$, it is important to establish certain linear relations among these $\pi_{i}^{\prime}$. We do this next.

Since $\bar{\pi}_{i}=0, i>c$, from (12) we see that for $i>c$

$$
\begin{aligned}
0 & =\sum_{j \geq 0} \pi_{j}^{\prime}\left(\begin{array}{c}
n-j \\
i-j
\end{array}\right)(1+y)^{j}(2 y)^{i-j} \\
& =\left(\begin{array}{c}
n \\
i
\end{array}\right)(2 y)^{i}+\theta^{i} \pi \prime_{i}+\sum_{j=c+1}^{i-1}\left(\begin{array}{c}
n-j \\
i-j
\end{array}\right) \theta^{j}(2 y)^{i-1} \pi \prime_{j} .
\end{aligned}
$$

Substituting in (13), we get

$$
\pi_{i}^{\prime}=2^{2 i-1} y\left(\begin{array}{c}
n \\
i
\end{array}\right)-\sum_{j=c+1}^{i-1}\left(\begin{array}{c}
n-j \\
i-j
\end{array}\right) 2^{2(i-j)} \pi{ }_{j} .
$$

Now, by substituting the values of $\bar{\pi}_{i}$ given in (14) in the relations for $\delta_{c}^{2}, \chi^{2}$ and $\delta_{c}^{+} \delta_{c}^{-}$from Theorem 2] and using elementary binomial identities (see [8] for example) we get the relations

$$
\delta_{c}^{2}+2^{c+1} \delta_{c}= \begin{cases}2^{2 c-1} y\left[1+(-1)^{s-1}\left(\begin{array}{c}
n-1 \\
c
\end{array}\right)\right] & (m=2 n), \\
2^{2 c-1} y\left[1+(-1)^{s-1}\left(\begin{array}{l}
n \\
c
\end{array}\right)\right] & (m=2 n+1),\end{cases}
$$




$$
\begin{gathered}
\chi^{2}=\left(\delta_{c}^{+}-\delta_{c}^{-}\right)^{2}=\theta^{n} \bar{\pi}_{c}=(-1)^{s-1} 2^{2 c-1} y\left(\begin{array}{l}
n \\
c
\end{array}\right) \quad(m \text { even, } k \text { even }), \\
\delta_{c}^{+} \delta_{c}^{-}+2^{c-1} \delta_{c}=2^{2 c-3} y\left[1+(-1)^{s}\left(\begin{array}{c}
n-1 \\
c-1
\end{array}\right)\right] \quad(m \text { even, } k \text { even }),
\end{gathered}
$$

From (18) and $\delta_{c}=\delta_{c}^{+}+\delta_{c}^{-}$, it follows trivially that

$$
\delta_{c} \delta_{c}^{+}=2^{2 c-3} y\left[1+(-1)^{s}\left(\begin{array}{c}
n-1 \\
c-1
\end{array}\right)\right]-2^{c-1} \delta_{c}+\left(\delta_{c}^{+}\right)^{2} \quad(m \text { even, } k \text { even }) .
$$

The ring structure of $B$ is thus given by

Proposition 8. Let $L=\left[1+(-1)^{s-1}(\underset{c}{[(m-1) / 2]})\right]$ and $M=\left[1+(-1)^{s}\left(\begin{array}{c}n-1 \\ c-1\end{array}\right)\right]$.

(i) For $m-k$ odd, as an abelian group

$$
B \approx Z \oplus Z y \oplus Z \delta_{c} \oplus Z \delta_{c} y
$$

with multiplication given by the table

\begin{tabular}{|c|c|c|}
\hline & $y$ & $\delta_{c}$ \\
\hline$y$ & $-2 y$ & $\delta_{c} y$ \\
\hline$\delta_{c}$ & $\delta_{c} y$ & $2^{c+1} \delta_{c}+2^{2 c-1} y L$ \\
\hline
\end{tabular}

As a ring, $B \approx Z\left[\delta_{c}, y\right] / I$, where $I$ is the ideal generated by $y^{2}+2 y$ and $\delta_{c}^{2}+2^{c+1} \delta_{c}-$ $2^{2 c-1} y L$.

(ii) For $m$ and $k$ both even

$$
B \approx Z \oplus Z y \oplus Z \delta_{c} \oplus Z \delta_{c}^{+} \oplus Z \delta_{c} y \oplus Z \delta_{c}^{+} y \oplus Z\left(\delta_{c}^{+}\right)^{2} \oplus Z\left(\delta_{c}^{+}\right)^{2} y
$$

with multiplication given by the table

\begin{tabular}{|c|c|c|c|}
\hline & $y$ & $\delta_{c}$ & $\delta_{c}^{+}$ \\
\hline$y$ & $-2 y$ & $y \delta_{c}$ & $y \delta_{c}^{+}$ \\
\hline$\delta_{c}$ & $y \delta_{c}$ & $-2^{c+1} \delta_{c}+2^{2 c-1} y L$ & $-2^{c-1} \delta_{c}+2^{2 c-3} y M+\left(\delta_{c}^{+}\right)^{2}$ \\
\hline$\delta_{c}^{+}$ & $y \delta_{c}^{+}$ & $-2^{c-1} \delta_{c}+2^{2 c-3} y M+\left(\delta_{c}^{+}\right)^{2}$ & $\left(\delta_{c}^{+}\right)^{2}$ \\
\hline
\end{tabular}

or, $B \approx Z\left[y, \delta_{c}, \delta_{c}^{+}\right] / J$, where $J$ is the ideal generated by $y^{2}+2 y, \delta_{c}^{2}+2^{c+1} \delta_{c}-$ $2^{2 c-1} y L$, and $\delta_{c} \delta_{c}^{+}+2^{c-1} \delta_{c}-2^{2 c-3} y M-\left(\delta_{c}^{+}\right)^{2}$.

Remark 3 . The remaing products, with $\left(\delta_{c}^{2}\right)^{2}$ and $y\left(\delta_{c}^{2}\right)$ are easily deduced in terms of the abelian group generators for $\mathrm{B}$ using the products given above.

7.2. Structure of $A$. As for $A$, from relation (16) (and proceeding as in [2]), we can choose a new basis for $A$, namely $\tau_{1}, \cdots, \tau_{s-3}, \rho_{1}, \rho_{2}, \rho_{3}$, such that $\operatorname{Res}\left(\tau_{i}\right)=0, i=$ $1, \cdots, s-3$, and, for $m=2 n+1$ and $k=2 s, \operatorname{Res}\left(\rho_{1}\right)=0, \operatorname{Res}\left(\rho_{2}\right)=\operatorname{Res}\left(\delta_{n}\right)$, $\operatorname{Res}\left(\rho_{3}\right)=b y$, while for $m=2 n$ and $k=2 s$ or $2 s-1, \operatorname{Res}\left(\rho_{1}\right)=\operatorname{Res}\left(\chi_{n}\right)$, $\operatorname{Res}\left(\rho_{2}\right)=\operatorname{Res}\left(\delta_{n}^{+}\right), \operatorname{Res}\left(\rho_{3}\right)=b y$.

In both cases $b=$ g.c.d. $\left\{2^{2 i-1}\left(\begin{array}{c}n \\ i\end{array}\right), i=c+1, \cdots,[m / 2]-2\right\}$, and we remind the reader that the formulae for $\operatorname{Res}\left(\delta_{n}\right), \operatorname{Res}\left(\chi_{n}\right)$, and $\operatorname{Res}\left(\delta_{n}^{+}\right)$were given in $\S 5$ Hence, from 6.2(2) we have, for $m=2 n$,

$$
\operatorname{Tor}_{A}^{*}(B ; Z) \approx \Lambda_{Z}^{*}\left[t_{1}, \cdots, t_{s-3}\right] \otimes_{Z} \operatorname{Tor}_{A^{\prime}}^{*}(B ; Z)
$$

with $\operatorname{dim} t_{i}=1, i=1, \cdots, s-3$, and $A^{\prime}=A /\left(\tau_{1}, \cdots, \tau_{s-3}\right)$, while, for $m=2 n+1$,

$$
\operatorname{Tor}_{A}^{*}(B ; Z) \approx \Lambda_{Z}^{*}\left[t_{1}, \cdots, t_{s-2}\right] \otimes_{Z} \operatorname{Tor}_{A^{\prime \prime}}^{*}(B ; Z)
$$

with $\operatorname{dim} t_{i}=1, i=1, \cdots, s-2$, and $A^{\prime \prime}=A /\left(\tau_{1}, \cdots, \tau_{s-3}, \rho_{1}\right)$. 
7.3. $K^{*}\left(X_{m, k}\right)$ for $m k$ even. We treat separately the three cases $m$ even, $m$ odd $k$ even and, $m$ odd $k$ odd, noting that in all cases $A, A^{\prime}$, and $A^{\prime \prime}$ are polynomial algebras so that the Koszul resolution is applicable.

7.3.1. $m$ even. Tor $_{A^{\prime}}^{*}(B ; Z)$ is the homology of the Koszul complex $\Lambda_{B}^{*}\left(x_{1}, x_{2}, x_{3}\right)$, where $d\left(x_{i}\right)=\operatorname{Res}\left(\rho_{i}\right), i=1,2,3$. As in [2, we take a new basis $u_{1}, u_{2}, u_{3}$ for $\Lambda_{B}^{*}\left(x_{1}, x_{2}, x_{3}\right)$ with $d\left(u_{1}\right)=2^{\alpha} y, 2^{\alpha}=g . c . d .\left\{2^{n-1}, b\right\} ; d\left(u_{2}\right)=2^{s-1} \delta_{c} ;$ and $d\left(u_{3}\right)=$ 0 . Hence for $k=2 s-1$

$$
\operatorname{Tor}_{A^{\prime}}^{*}(B ; Z) \approx \Lambda_{Z}^{*}\left[z_{1}, z_{2}, z_{3}, z_{4}\right] \otimes Z\left[y, \delta_{c}\right] / I
$$

and for $k=2 s$

$$
\operatorname{Tor}_{A^{\prime}}^{*}(B ; Z) \approx \Lambda_{Z}^{*}\left[z_{1}, z_{2}, z_{3}, z_{4}\right] \otimes Z\left[y, \delta_{c}, \delta_{c}^{+}\right] / J
$$

with $y, \delta_{c}$ and $\delta_{c}^{+}$of degree 0 and, $z_{1}, z_{2}, z_{3}, z_{4}$ of degree 1 given by, $z_{1}=(y+2) u_{1}$, $z_{2}=-2^{n+c-2-\alpha} L u_{1}+\left(\delta_{c}+2^{c+1}\right) u_{2}, z_{4}=-2^{s-1-r} \delta_{c} u_{1}+2^{\alpha-r} y u_{2}$ and $z_{3}=u_{3}$.

For $k=2 s-1, I$ is the ideal generated by: $y^{2}+2 y ; \delta_{c}^{2}+2^{c+1} \delta_{c}-2^{2 c-1} y L ; 2^{s-1} \delta_{c}$; $2^{\alpha} y ;(y+2) z_{4}-2^{s-1-r} \delta_{c} z_{1} ; 2^{r} z_{4}$, where $r=\min \{\alpha, s-1\} ; z_{1} y ; z_{1} z_{4} ; z_{2} z_{4} ; z_{2} \delta_{c}-$ $2^{2 c-1+r-\alpha} L z_{4} ;$ and $\delta_{c} z_{4}+2^{c+1} z_{4}-2^{\alpha-r} y z_{2}$.

For $k=2 s, J$ is the ideal generated by the same elements given in $I$ and the element $\delta_{c} \delta_{c}^{+}+2^{c-1} \delta_{c}-2^{2 c-3} y M-\left(\delta_{c}^{+}\right)^{2}$.

Therefore we have

Theorem 4. The Hodgkin spectral sequence for $X_{2 n, k}$ collapses, and so, as graded algebras,

$$
\begin{aligned}
K^{*}\left(X_{2 n, 2 s-1}\right) & \approx \Lambda_{Z}^{*}\left[t_{1}, \cdots, t_{s-3}, z_{1}, z_{2}, z_{3}, z_{4}\right] \otimes Z\left[y, \delta_{c}\right] / I \\
K^{*}\left(X_{2 n, 2 s}\right) & \approx \Lambda_{Z}^{*}\left[t_{1}, \cdots, t_{s-3}, z_{1}, z_{2}, z_{3}, z_{4}\right] \otimes Z\left[y, \delta_{c}, \delta_{c}^{+}\right] / J
\end{aligned}
$$

where $I$ and $J$ are the ideals generated by the above elements, except that $z_{1} z_{4}$ and $z_{2} z_{4}$ are replaced by $z_{1} z_{4}+\lambda, \quad z_{2} z_{4}+\mu$, for some $\lambda, \mu \in Z\left[y, \delta_{c}\right]$ if $k$ odd, or by $\lambda, \mu \in Z\left[y, \delta_{c}, \delta_{c}^{+}\right]$if $k$ even.

Proof. This follows from (11), (18), (23), (24), (25) (or (26) for $k=2 s$ ), Theorem 1 Note 2 of 86 and $\S 6$ of [2]. As for the elements $z_{i} z_{4}, i=1,2$, they are in Tor $^{2}=E_{2}^{\infty}=\tilde{F}^{2} / \tilde{F}^{0}$. Since $z_{i} z_{4}=0$ in Tor ${ }^{*}$, we have $z_{i} z_{4} \in \tilde{F}^{0}\left(K^{*}\right) \subset K^{0}$. Hence, in $K^{0}, z_{i} z_{4}=a_{i} y+b_{i} \delta_{c}+c_{i} \delta_{c} y, a_{i}, b_{i}, c_{i} \in Z, i=1,2$ for $k=2 s-1$ (similarly for $k$ even with the extra terms added).

7.3.2. $m$ odd, $k$ even. $\operatorname{Tor}_{A^{\prime \prime}}^{*}(B ; Z)$ is the homology of the Koszul complex $\Lambda_{B}^{*}\left(x_{1}, x_{2}\right)$ :

$$
0 \longrightarrow B \longrightarrow B \oplus B \stackrel{d}{\longrightarrow} B \longrightarrow 0
$$

where $d\left(x_{1}\right)=\operatorname{Res}\left(\rho_{3}\right)=b y$ and $d\left(x_{2}\right)=\operatorname{Res}\left(\delta_{n}\right)=2^{s-1}(2+y) \delta_{c}+2^{n-1} y$. Then, in $\operatorname{Tor}_{A^{\prime \prime}}^{0}(B ; Z)$ we have $b y=0$ and $2^{s-1}(2+y) \delta_{c}+2^{n-1} y=0$. Hence, by multiplying the second equation by $y$, it follows that $2^{n} y=0$. Thus $2^{\alpha} y=0$, where $2^{\alpha}=$ g.c.d. $\left\{2^{n}, b\right\}$.

For $i \geq 1, H_{i}\left(\Lambda_{B}^{*}\right)$ may be computed by hand or using a suitable software such as Maple (Maple V Release 4, Waterloo Maple Inc., June 1996, Gröbner package). A set of generators of $H_{1}$ is, $z_{1}=\left(-2^{n+c-r}-2^{n-1-r} \delta_{c}\right) x_{1}+\left(2^{c+1-r} b+2^{-r} b \delta_{c}\right) x_{2}$, $r=\min \{n-1, c+1\}, z_{2}=(2+y) x_{1}$, and $z_{3}=2^{n-\alpha} x_{1}+2^{-\alpha} b y x_{2}$. 
From this and by calculating $H_{i}, i>1$ from the Koszul resolution, it is not hard to verify that as graded algebras

$$
\operatorname{Tor}_{A}^{*}(B ; Z) \approx \Lambda_{Z}^{*}\left[z_{1}, z_{2}, z_{3}\right] \otimes Z\left[y, \delta_{c}\right] / I
$$

with $z_{1}, z_{2}, z_{3}$ of degree $1, y$ and $\delta_{c}$ of degree 0 , and $I$ the ideal generated by $y^{2}+2 y ; z_{2} z_{3} ; y z^{2} ; \delta_{c}^{2}+2^{c+1} \delta_{c}-2^{2 c-1} y L ; 2^{\alpha} y ; 2^{s-1}(2+y) \delta_{c}+2^{n-1} y ; z_{2} z_{3} ; 2^{r} \delta_{c} z_{1}$ $+2^{2 c-2+\alpha} L y z_{3} ; 2^{r} y z_{1}+2^{n-1} \delta_{c} z_{2}+2^{\alpha}\left(2^{c} y-\delta_{c}\right) z_{3} ;-2^{n-\alpha} z_{2}+(2+y) z_{3} ; 2^{\alpha} z_{3}-$ $\left(2^{n-1}+2^{s-1} \delta_{c}\right) z_{2}$.

Hence we have

Theorem 5. The Hodgkin spectral sequence for $X_{2 n+1,2 s}$ collapses, and so, as graded algebras,

$$
K^{*}\left(X_{2 n+1,2 s}\right) \approx \Lambda_{Z}^{*}\left[t_{1}, \cdots, t_{s-2}, z_{1}, z_{2}, z_{3}\right] \otimes Z\left[y, \delta_{c}\right] / I,
$$

where $I$ is the ideal generated by the elements listed in (27).

Remark 4 . The element $y+1 \in K^{*}\left(X_{m, k}\right)$ can be identified with the complexified Hopf bundle $c \xi_{m, k}$ over $X_{m, k}$ (see [2]). Then it follows from the preceding results that for $m k$ even the order of $c \xi_{m, k}$ is

$$
2^{\alpha(m, k)}=\text { g.c.d. }\left\{2^{[(m-1) / 2]}, 2^{2 i-1}\left(\begin{array}{c}
n \\
i
\end{array}\right), i=c+1, \cdots,[(m-3) / 2]\right\} .
$$

\section{8. $K^{*}\left(X_{m, k}\right)$ FOR $m k$ ODD}

When $m k$ is odd, various things go wrong. First, the Pontrjagin classes do not make sense, so we are obliged to work with the exterior powers $\bar{\lambda}_{i}=\bar{\Lambda}^{i}-\left(\begin{array}{c}2 c \\ i\end{array}\right)$ instead (recalling that we are writting $\bar{\Lambda}[t]$ for the $\Lambda[t]$ corresponding to $R H_{m, k}[t]$ in order to distinguish the $\Lambda[t]$ in $\operatorname{RSpin}(2 n+1)[t])$. Second, there is the more serious problem that $R H$ is no longer a free $Z\left[\lambda_{1}, \cdots, \lambda_{c}\right]$-module, so that change of rings cannot be used.

We shall therefore content ourselves with calculating $K^{0}\left(X_{m, k}\right)$ and, in particular, the order of $y$ and $\delta_{c}$ in $K^{*}\left(X_{m, k}\right)$. The relevant complex is $\Lambda_{R H}^{*}\left[x_{1}, \cdots, x_{n}\right]$, which is given by

$$
0 \rightarrow \Lambda_{R H}^{n}\left(x_{1}, \cdots, x_{n}\right) \rightarrow \cdots \rightarrow \Lambda_{R H}^{1}\left(x_{1}, \cdots, x_{n}\right) \rightarrow \Lambda_{R H}^{0}\left(x_{1}, \cdots, x_{n}\right)=R H,
$$

where $d\left(x_{i}\right)=\operatorname{Res}\left(\lambda_{i}\right)=\lambda_{i}^{\prime}(0<i<n)$ and $d\left(x_{n}\right)=\operatorname{Res}\left(\delta_{n}\right)=2^{s} \delta_{c}$. Then, since $H_{0}\left(\Lambda_{R H}^{*}\right)=R H / \operatorname{Im} d$, we have

$$
H_{0}\left(\Lambda_{R H}^{*}\right)=Z\left[\lambda_{1}, \cdots, \lambda_{c-1}, \delta_{c}, y\right] /\left(\lambda_{1}^{\prime}, \cdots, \lambda_{n-1}^{\prime}, 2^{s} \delta_{c}, y^{2}+2 y, y \delta_{c}+2^{c} y\right) .
$$

In $R H$ we have, recalling Lemma 1, Cor. 1, and 4.3, Cor. 1, that $\bar{\Lambda}^{j}=\theta \bar{\Lambda}^{2 c-j}$ for all $j$ and $\bar{\Lambda}^{c}=\bar{\Delta}_{c}^{2}-(1+\theta)\left(1+\bar{\Lambda}^{1}+\ldots+\bar{\Lambda}^{c-1}\right)$ (Theorem 2). Also note that $y \bar{\Delta}_{c}=(\theta-1) \bar{\Delta}_{c}=\bar{\Delta}_{c}-\bar{\Delta}_{c}=0$.

Let $\lambda^{\prime}[t]=\Lambda^{\prime}[t]-(1+t)^{2 n+1}=\sum_{i>0} \lambda_{i}^{\prime} t^{i}\left(\lambda_{i}^{\prime}=\chi_{i} \lambda^{\prime}[t]\right.$, where $\chi_{i}$ stands for "coefficient of $t^{i}$ " as usual). Using this and applying (9), we have

$$
\lambda^{\prime}[t]=(1+t \theta)^{2 s+1}(\bar{\Lambda}[t]-f(t)),
$$

where $f(t)=F(\theta, t)$, with (by definition)

$$
F(z, t)=\frac{(1+t)^{2 n+1}}{(1+z t)^{2 s+1}} .
$$


Thus

$$
\chi_{i}(1+t \theta)^{-2 s-1} \lambda^{\prime}[t]=\chi_{i}(\bar{\Lambda}[t]-f(t))=\bar{\Lambda}^{i}-\chi_{i} f(t), \quad i>0 .
$$

Now in $H_{0}\left(\Lambda_{R H}^{*}\right)$ we have $\lambda_{i}^{\prime}=0,0<i<n$. Since $\chi_{i}(1+t \theta)^{-2 s-1} \lambda^{\prime}[t]$ is a $Z[\theta]$-linear combination of $\lambda_{1}^{\prime}, \cdots, \lambda_{n-1}^{\prime}$, from (28) we have

$$
\bar{\Lambda}^{i}-\chi_{i} f(t)=0, \quad 0<i<n .
$$

But in $R H, \bar{\Lambda}^{c+i}=\theta \bar{\Lambda}^{c-i}, i \geq 0$. Substituting this in (29), we have, in $H_{0}\left(\Lambda_{R H}^{*}\right)$,

$$
\left(\chi_{c+i}-\theta \chi_{c-i}\right) f(t)=0, \quad i \geq 0 .
$$

In particular,

$$
y \chi_{c} f(t)=0 .
$$

Our next objective is to replace the relations (30) and (31) by the single relation by, where

$$
b=\text { g.c.d. }\left\{2^{2 c}\left(\begin{array}{l}
n \\
c
\end{array}\right), 2^{2 i-1}\left(\begin{array}{c}
n \\
i
\end{array}\right), i=c+1, \cdots, n-1\right\} .
$$

For $i \geq 0,\left(\chi_{c+i}-z \chi_{c-i}\right) F(z, t)$ is a polynomial, $h_{i}(z)$, say. Clearly, $h_{i}(1)=$ $\left(\chi_{c+i}-\chi_{c-i}\right)(1+t)^{2 c}=\left(\begin{array}{c}2 c \\ c+i\end{array}\right)-\left(\begin{array}{c}2 c \\ c-i\end{array}\right)=0$. Since $h_{i}(1)=h_{i}(-1)$ modulo 2 and $\theta^{2}=1$,

$$
\begin{aligned}
h_{i}(\theta) & =\frac{1}{2}\left(h_{i}(1)+h_{i}(-1)\right)+\frac{\theta}{2}\left(h_{i}(1)-h_{i}(-1)\right) \\
& =\frac{1+\theta}{2} h_{i}(1)+\frac{1-\theta}{2} h_{i}(-1)=0-\frac{1}{2} y h_{i}(-1) .
\end{aligned}
$$

Now $h_{i}(-1)=\left(\chi_{c+i}+\chi_{c-i}\right) F_{s, c}$ where $F_{s, c}=(1+t)^{2 c}\left(\frac{1+t}{1-t}\right)^{2 s+1}$. It follows that

$$
\begin{aligned}
y \chi_{c} f(t) & =(\theta-1) \chi_{c} f(t)=-(1-\theta) \chi_{c} F(\theta, t) \\
& =-\left(\chi_{c}-\theta \chi_{c}\right) F(\theta, t)=-h_{0}(\theta)=\frac{1}{2} y h_{0}(-1)=y \chi_{c} F_{s, c},
\end{aligned}
$$

and also

$$
\left(\chi_{c+i}-\theta \chi_{c-i}\right) f(t)=h_{i}(\theta)=-\frac{1}{2} y h_{i}(-1)=-\frac{1}{2}\left(\chi_{c+i}+\chi_{c-i}\right) F_{s, c} .
$$

Lemma 7. With the above notation,

(i) $\chi_{c} F_{s, c}=2^{2 c}\left(\begin{array}{c}s+c \\ c\end{array}\right)$, all $s \geq 0$, all $c \geq 0$.

(ii) For all $i>0$ we have $\left(\chi_{c+i}+\chi_{c-i}\right) F_{s, c}=\chi_{c+i} F_{s, c+i}-\left(\begin{array}{c}2 i \\ i\end{array}\right) \chi_{c} F_{s, c}+Z$-linear combination of $\left(\chi_{c+j}+\chi_{c-j}\right) F_{s, c}(0<j<i)$.

Proof. (i) By induction on $n=s+c$ starting with $n=0$ and using

(a) $\chi_{c} F_{s, c}=\chi_{c} F_{s-1, c}+4 \chi_{c-1} F_{s, c-1}$ (this follows from $F_{s, c}-F_{s-1, c}=4 t F_{s, c-1}$ ),

(b) $\chi_{0} F_{s, 0}=1$, all $s \geq 0$,

(c) $\chi_{c} F_{0, c}=\chi_{c}\left(\frac{(1+t)^{2 c+1}}{1-t}\right)=2^{2 c}, \forall c \geq 0$ (this follows using $\frac{1}{1-t}=\sum_{i \geq 0} t^{i}$ ).

(ii) We have, for $i>0$,

$$
(1+t)^{2 i}=1+t^{2 i}+\left(\begin{array}{c}
2 i \\
i
\end{array}\right) t^{i}+\sum_{0<j<i}\left(\begin{array}{c}
2 i \\
j
\end{array}\right) t^{j}\left(1+t^{2(i-j)}\right) .
$$

Hence $\left(\chi_{c+i}+\chi_{c-i}\right) F_{s, c}=\chi_{c+i}\left(1+t^{2 i}\right) F_{s, c}$ and the result follows. 
From the lemma we deduce (respectively from (i), (ii))

1. $y \chi_{c} f=2^{2 c}\left(\begin{array}{c}s+c \\ c\end{array}\right) y$.

2. For $i>0, \frac{1}{2} h_{i}(-1)=2^{2(c+i)-1}\left(\begin{array}{c}s+c+i \\ i\end{array}\right)-\frac{1}{2}\left(\begin{array}{c}2 i \\ i\end{array}\right) 2^{2 c}\left(\begin{array}{c}s+c \\ c\end{array}\right)+Z$-linear combination of $\frac{1}{2} h_{j}(-1)(0<j<i)$.

An easy inductive argument (using Pascal's triangle) now shows that

$$
\begin{aligned}
& \text { g.c.d. }\left\{2^{2 c}\left(\begin{array}{c}
s+c \\
c
\end{array}\right), \frac{1}{2} h_{i}(-1)(0<i<n-c)\right\} \\
= & \text { g.c.d. }\left\{2^{2 c}\left(\begin{array}{c}
s+c \\
c
\end{array}\right), 2^{2(c+i)-1}\left(\begin{array}{c}
s+c \\
c+i
\end{array}\right)(0<i<n-c)\right\} .
\end{aligned}
$$

Writing $b$ for this, we conclude that the relations $y \chi_{c} f(t),\left(\chi_{c+i}-\theta \chi_{c-i}\right) f(t)(0<$ $i<n-c)$ may be replaced by the single relation by.

The next item on the agenda is the relation involving $\bar{\Delta}_{c}^{2}$. In $H_{0}\left(\Lambda_{R H}^{*}\right)$ we have

$$
\begin{aligned}
\bar{\Delta}_{c}^{2} & =(1+\theta)\left(1+\bar{\Lambda}^{1}+\cdots+\bar{\Lambda}^{c-1}\right)+\bar{\Lambda}^{c} \\
& =(1+\theta)\left(1+\chi_{1}+\cdots+\chi_{c-1}\right) f(t)+\chi_{c} f(t) .
\end{aligned}
$$

But

$$
\begin{aligned}
(1+\theta) \chi_{i} f(t) & =\chi_{i}(1+\theta) f(t)=\chi_{i}(1+\theta)(1+t)^{2 c}\left(\frac{1+t}{1+\theta t}\right)^{2 s+1} \\
& =\chi_{i}(1+\theta)(1+t)^{2 c}
\end{aligned}
$$

since $\theta(1+\theta)=(1+\theta)$ and using the binomial theorem. Substituting this in the relation $(32)$ for $\bar{\Delta}_{c}^{2}$, we have

$$
\begin{aligned}
\bar{\Delta}_{c}^{2} & =(1+\theta)\left(1+\chi_{1}+\cdots+\chi_{c-1}\right)(1+t)^{2 c}+\chi_{c} f(t) \\
& =(1+\theta) \sum_{i=0}^{c-1}\left(\begin{array}{c}
2 c \\
c
\end{array}\right)+\chi_{c} f(t)=(2+y) \frac{1}{2}\left[2^{2 c}-\left(\begin{array}{c}
2 c \\
c
\end{array}\right)\right]+\chi_{c} f(t) .
\end{aligned}
$$

Now

$$
\begin{gathered}
\chi_{c} f=\chi_{c} F(\theta, t)=\chi_{c}\left(\frac{F(1, t)+F(-1, t)}{2}\right)+\theta \chi_{c}\left(\frac{F(1, t)-F(-1, t)}{2}\right) \\
=\frac{1}{2}\left(\left(\begin{array}{c}
2 c \\
c
\end{array}\right)+2^{2 c}\left(\begin{array}{l}
n \\
c
\end{array}\right)\right)+\frac{1}{2}\left(\left(\begin{array}{c}
2 c \\
c
\end{array}\right)-2^{2 c}\left(\begin{array}{l}
n \\
c
\end{array}\right)\right) \theta
\end{gathered}
$$

(using Lemma 7 for $F(-1, t)$ ). Hence from $(33)$ in $H_{0}\left(\Lambda_{R H}^{*}\right)$ we have

$$
\bar{\Delta}_{c}^{2}=2^{2 c-1} y\left[1-\left(\begin{array}{l}
n \\
c
\end{array}\right)\right]+2^{2 c},
$$

or, equivalently,

$$
\delta_{c}^{2}+2^{c+1} \delta_{c}=2^{2 c-1} y\left[1-\left(\begin{array}{l}
n \\
c
\end{array}\right)\right]
$$

Thus,

$$
K^{0}\left(X_{m, k}\right)=Z\left[\bar{\Delta}_{c}, y\right] /\left(y^{2}+2 y, \bar{\Delta}_{c} y, 2^{s} \delta_{c}, b y, \bar{\Delta}_{c}^{2}-D\right),
$$

where $D=2^{2 c}+2^{2 c-1}\left\{1-\left(\begin{array}{l}n \\ c\end{array}\right)\right\} y$.

Let $R$ be the ring $Z[y] /\left(y^{2}+2 y, b y\right)$. Then

$$
K^{0}\left(X_{m, k}\right)=R\left[\bar{\Delta}_{c}\right] /\left(y \bar{\Delta}_{c}, 2^{s} \delta_{c}, \bar{\Delta}_{c}^{2}-D\right) .
$$

An easy calculation shows that $R=Z \oplus Z_{b} y\left(Z_{b}\right.$ being $\left.Z / b\right)$. 
Consider the inclusion $R+R \bar{\Delta}_{c}$ in $R\left[\bar{\Delta}_{c}\right]$. This induces an epimorphism $R+$ $R \bar{\Delta}_{c} \rightarrow K^{0}\left(X_{m, k}\right)$ (because of the relations $y \bar{\Delta}_{c}, \bar{\Delta}_{c}^{2}-D$ ). Now $r_{0}+r_{1} \bar{\Delta}_{c}$ lies in the kernel if and only if $r_{0}+r_{1} \bar{\Delta}_{c}=F y \bar{\Delta}_{c}+G\left(2^{s} \bar{\Delta}_{c}-2^{n}\right)+H\left(\bar{\Delta}_{c}^{2}-D\right)$, where $r_{0}, r_{1} \in R$ and $F, G, H \in R\left[\bar{\Delta}_{c}\right]$. Writing $F=F_{0}\left(\bar{\Delta}_{c}^{2}\right)+\bar{\Delta}_{c} F_{1}\left(\bar{\Delta}_{c}^{2}\right)$ and similarly for $G$ (note that any $F, G \in R\left[\bar{\Delta}_{c}\right]$ can be written this way), we see that the expression

$$
\begin{gathered}
\left(F_{0}\left(\bar{\Delta}_{c}^{2}\right)+\bar{\Delta}_{c} F_{1}\left(\bar{\Delta}_{c}^{2}\right)\right) \bar{\Delta}_{c} y+\left(G_{0}\left(\bar{\Delta}_{c}^{2}\right)+\bar{\Delta}_{c} G_{1}\left(\bar{\Delta}_{c}^{2}\right)\right)\left(2^{s} \bar{\Delta}_{c}-2^{n}\right) \\
-\left\{F_{0}(D) \bar{\Delta}_{c} y+D F_{1}(D) y+2^{s} G_{0}(D) \bar{\Delta}_{c}-2^{n} \bar{\Delta}_{c} G_{1}(D)-2^{n} G_{0}(D)+2^{s} D G_{1}(D)\right\}
\end{gathered}
$$

is divisible by $\bar{\Delta}_{c}^{2}-D$. Thus, for a suitable choice of $H$,

$$
r_{0}+r_{1} \bar{\Delta}_{c}=F_{0}(D) \bar{\Delta}_{c} y+G_{0}(D)\left\{2^{s} \bar{\Delta}_{c}-2^{n}\right\}+G_{1}(D)\left\{2^{s} D-2^{n} \bar{\Delta}_{c}\right\}
$$

(here we used the fact that $D y=0$ in $R$, which follows easily from $b y=0$ ).

Now $F_{0}(D), G_{0}(D), G_{1}(D)$ are arbitrary elements of $R$. Hence the kernel above is generated over $Z$ by $\bar{\Delta}_{c} y, \bar{\Delta}_{c} y^{2}, 2^{s} \bar{\Delta}_{c}-2^{n}, 2^{s} \bar{\Delta}_{c} y-2^{n} y, 2^{s} D-2^{n} \bar{\Delta}_{c}$ and $2^{s} D y-2^{n} \bar{\Delta}_{c} y$, or, equivalently, by $\Delta_{c} y, 2^{s} \delta_{c}, 2^{n} y, 2^{s} D-2^{n} \bar{\Delta}_{c}$, that is, by $\bar{\Delta}_{c} y, 2^{s} \delta_{c}, 2^{n} y, 2^{s+2 c-1}\left\{1-\left(\begin{array}{l}n \\ c\end{array}\right)\right\} y=2^{n+c-1}\left[1-\left(\begin{array}{l}n \\ c\end{array}\right)\right] y$. But the last term is zero even if $s=0$ (for then $\left(\begin{array}{l}n \\ c\end{array}\right)=1$ ). Thus,

$$
\begin{aligned}
K^{0}\left(X_{m, k}\right) & =\left(R+R \bar{\Delta}_{c}\right) /\left(2^{s} \delta_{c}, \Delta_{c} y, 2^{n} y\right)=\left(R+Z \bar{\Delta}_{c}\right) /\left(2^{s} \delta_{c}, 2^{n} y\right) \\
& =\left(Z+Z_{b} y+Z \delta_{c}\right) /\left(2^{s} \delta_{c}, 2^{n} y\right)=Z+Z_{2^{\alpha}} y+Z_{2^{s}} \delta_{c},
\end{aligned}
$$

where

$$
2^{\alpha}=\text { g.c.d. }\left\{2^{n} ; 2^{2 c}\left(\begin{array}{c}
n \\
c
\end{array}\right) ; 2^{2(c+i)-1}\left(\begin{array}{c}
n \\
c+i
\end{array}\right), 0<i<n-c\right\} .
$$

Furthermore, $y^{2}=-2 y, y \delta_{c}=-2^{c} y$, and $\delta_{c}^{2}=-2^{c+1} \delta_{c}+2^{2 c-1}\left[1-\left(\begin{array}{l}n \\ c\end{array}\right)\right] y$.

We end by observing that:

1. The generators occurring in Theorems 4 and 5 may be described geometrically via the $\alpha$ and $\beta$ constructions, as in [10] and [12].

2. Using the Gysin sequence, $K^{*}\left(X_{2 n, 2 s}\right)$ may be deduced from $K^{*}\left(X_{2 n, 2 s-1}\right)$ as in [7, but $K^{*}\left(X_{2 n+1,2 s+1}\right)$ cannot be similarly deduced, as $X_{2 n+1,2 s+1}$ is nonorientable.

3. In the special cases $X_{2 n, 2 n-1}=P O(2 n)$ and $X_{m, 1}=R P^{m-1}$ these results reduce to those of [9], 10] and [1]. For $X_{4 n, 2 k-1}$ they agree with [2].

\section{REFERENCES}

1. Adams, J.F. - Vector Fields on Spheres, Ann. of Math., 75 (1962), 603-632. MR 25:2614

2. Antoniano, E., Gitler, S., Ucci, J. and Zvengrowski, P. - On the $K$-theory and parallelizability of projective Stiefel manifolds, Boletin de la Sociedad Matemática Mexicana, (1986), 29-116. MR 89g:57058

3. Barufatti, N. - Obstructions to immersions of projective Stiefel manifolds, Contemp. Math., AMS, vol. 161 (1994), pp. 281-287. MR 95c:57049

4. Bröcker, T. and tom Dieck, T. -Representations of compact Lie groups, G.T.M., SpringerVerlag (1985). MR 86i:22023

5. Cartan, H. and Eilenberg, S. - Homological Algebra, Princeton University Press (1956). MR 17:1040e

6. Gitler, S. and Handel, D. - The projective Stiefel manifolds I, Topology 7, (1968), 39-46. MR 36:3373a

7. Gitler, S. and Lam, K. Y. - The K-theory of Stiefel manifolds, Lecture Notes in Math., vol. 168, Springer-Verlag, 168 (1970), pp. 35-66. MR 43:1223

8. Gould, H. W. - Combinatorial Identities, Morgantown Printing, Morgantown, W. Va. (1972). MR 50:5879 
9. Held, R. P. and Suter, U. - Stable vector bundles over the projective orthogonal groups, Comm. Math. Helv., 50 (1975), 93-114. MR 51:4246

10. Hodgkin, L. - The equivariant Künneth theorem in K-theory, Lectures Notes in Math., vol. 496, Springer -Verlag, 496 (1975), pp. 1-101. MR 57:17645

11. Husemoller, D. - Fibre Bundles, McGraw-Hill, New York (1966). MR 37:4821

12. Roux, A. - Application de la suite spectrale d'Hodgkin au calcul de la K-théorie des variétés de Stiefel, Bull. Soc. Math. France, 99 (1971), 345-368. MR 47:1057

Instituto Politécnico, UerJ, Caixa Postal 97282, CeP: 28601-970, Nova Friburgo, RJ, BRASIL

E-mail address: nelza@iprj.uerj.br

PUC-RJ, Departamento de Matemática, R. Marquês de São Vicente, 225, Gávea, Rio DE JANEIRo, RJ, Brasil, CEP:22453-900

E-mail address: derek@mat.puc-rio.br 